\title{
Substructures in Abell clusters of galaxies ${ }^{\star}$
}

\author{
P. Flin and J. Krywult
}

Pedagogical University, Institute of Physics, ul. Swietokrzyska 15, 25-406 Kielce, Poland

e-mail: sfflin@cyf-kr.edu.pl

Received 10 July 2005 / Accepted 18 November 2005

\section{ABSTRACT}

Aims. We present the results of substructure detection on a large sample of Abell clusters.

Methods. We apply the wavelet transform to positions of galaxies in 183 Abell clusters. The significance of the substructuring detected was determined using Monte Carlo simulations on sets of 1000 randomly generated distributions of galaxies for each cluster and wavelet scale.

Results. 62 of the investigated clusters are strongly substructured; the frequency of occurrence of substructure is 0.34 . The investigated clusters were classified as unimodal, bimodal or complex systems. The clusters were divided into clusters having substructures in the field of the cluster $S_{\mathrm{f}}$ or in the core region $\mathrm{S}_{\mathrm{c}}$. The Kolmogorov-Smirnov test show no significant differences at the level $\alpha=0.05$ in the distribution of clusters having $S_{c}$ and $S_{f}$ substructures with redshift. Using a $\chi^{2}$ test we found no correlation between the existence of subclustering and the morphological type of galaxy clusters.

Key words. galaxies: clusters: general

\section{Introduction}

The presence or absence of substructures in galaxy clusters is very important for the study of cluster formation and evolution. Over the past two decades, substructures have been detected in a significant fraction of galaxy clusters. The existence of subclustering suggests that clusters are dynamically young objects. This allows one to test cosmological models (Thomas et al. 1998; Knebe \& Muller 2000). The frequency of substructures in clusters was used in the past to evaluate the density parameter $\Omega_{\mathrm{o}}$ (Richstone et al. 1992; Kauffmann \& White 1993). Moreover, the properties of galaxies in clusters depend on their environment. In clusters, morphology segregation is found among galaxies both in substructures and outside them (Biviano et al. 2002). Similar results based on the APM cluster sample were obtained by Plionis (2001). He found that in the analyzed sample of data, dynamically young clusters are spatially much more clustered.

Kolokotronis et al. (2002) investigated a sample of 22 rich galaxy clusters, finding a strong correlation between the optical (APM) and X-ray (ROSAT) morphological parameters. Their work led to the conclusion that in both parts of the spectrum it is possible to identify the dynamical state of a cluster correctly. Important results were obtained by Schuecker et al. (2001). Analyzing X-ray surface brightness distribution of 470 clusters they found a substructure-density relation. 52\%

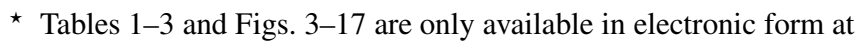
http://www.edpsciences.org of studied clusters had substructures in an aperture radius of $1 \mathrm{Mpc}$.

The analysis of cluster ellipticity (Plionis 2002) revealed correlation with cluster redshift. Cluster ellipticity increases with redshift for the investigated sample with $z<0.18$, see however Floor et al. (2003), Flin et al. (2004) and Rahman et al. (2004).

By constructing radial number-density profiles for about 100 clusters, Baier $(1979,1983)$ and Baier \& Mai $(1977,1978)$ concluded that as many as $80 \%$ of the clusters they studied had subclustering. Based on the projected distribution of galaxies, using surface density contour maps for 65 rich galaxy clusters, Geller \& Beers (1982) found that substructures are statistically significant in $40 \%$ of these cases. The same percentage of subclustering was reported by Dressler \& Shectman (1988) from 3D data (radial velocities of galaxies). West et al. (1988) reported a lower percentage of subclustering, finding little significant subclustering in the sample studied. Using both projected distributions and radial velocities of galaxies, West \& Bothun (1990) found structures in the outer parts of clusters. Krywult (1997) applied the symmetry and separation tests to positional data of 18 clusters and concluded that $32 \%$ of these clusters show substructures. Using a number of statistical tests on 33 clusters, Bird (1993) showed that depending on the method applied, from $10 \%$ to $40 \%$ have statistically significant substructures. Escalera et al. (1994) applied the wavelet analysis to 16 clusters classified by them as unimodal or bimodal. More recent studies were carried out by Kriessler \& Beers (1997), 
using the adaptive kernel technique for 56 clusters. They concluded that $57 \%$ of these clusters show substructures. A wavelet analysis of 18 Abell clusters (Krywult et al. 1999) showed the existence of substructures in $50 \%$ of cases. Using four different methods, Krywult \& Flin (2000) found that statistically significant subclustering is present in $40 \%$ of the investigated clusters.

A comparison of our work with other recent studies indicates a good correspondence between the fraction of substructures detected in 2D and 3D data. Thus, the analysis of substructures in galaxy clusters based on projected, i.e. 2D data, can reveal a statistically correct percentage of subclustering, disregarding some differences for individual objects (Flin \& Krywult 2002).

We present the results obtained from a large sample, which can be useful for comparison with other data. Our clusters contain objects within the standard 3 mag range and a visual verification of star/galaxy separation has been performed. We use the parameters $h=0.75$ and $q=0.5$.

Observational data are presented in Sect. 2, with the method applied, i.e. the wavelet analysis, given in Sect. 3. Section 4 presents the results. In Sect. 5 we discuss the individual clusters. Section 6 provides a conclusion.

\section{Observational data}

There are two sources of our data. The first is our previous papers (Trévese et al. 1992, 1997; Flin et al. 1995; Flin et al. 2000) derived from 10-inch photographic plates taken with the 48inch Palomar Schmidt Telescope (Hickson 1977). The emulsions employed were Kodak 127-02 or Kodak 098-04, both used with a 2 mm Schott RG-1 glass filter, which corresponds to the red photographic $F$-band of Oemler (1974). The plates were calibrated using the Palomar spot sensitometer.

The fields containing clusters originally selected by Hickson (1977), as well as some additional Abell (1989) clusters clearly visible on the plates, were scanned in the transparency mode using a PDS $1010 \mathrm{G}$ micro-densitometer, producing a digital image for each cluster field with a pixel size from 15 to $20 \mu \mathrm{m}$, according to the noise level of the plate and the cluster distance. Objects were automatically detected and magnitude values computed in many circular apertures, producing a magnitude profile from which the objects were automatically classified as point-like or diffuse.

Total magnitudes $m_{\mathrm{T}}$ were computed from the flux integrated in an aperture of radius $R_{1}=1.5 r_{1}$, where $r_{1}$ is the first momentum of intensity distribution (see Trévese et al. 1992).

With the above definition, total magnitudes correspond on average to the magnitude $m_{\text {iso }}$ computed in a circular aperture determined by the isophote $\mu=24 \mathrm{mag} \operatorname{arcsec}^{-2}$, with the advantage that $r_{1}$ is less noisy than the corresponding isophotal radius (see Flin et al. 1995). Magnitude-zero points are taken from the literature (Hoessel et al. 1980; Hoessel \& Schneider 1985; Sandage \& Perlmuter 1991; Peterson 1970; Gunn \& Oke 1975; Bothun et al. 1985; Murphy et al. 1983).

The majority of galaxy catalogues were obtained by applying FOCAS package (Jarvis \& Tyson 1981) to the Digitized
Sky Survey, which is the second source of our data. The essential difference between this and other samples is the visual checking of all objects classified as galaxies when automatic procedures were applied.

We take into account only well separated Abell clusters with clearly visible galaxies, which allows us to perform the visual verification of the automatic star/galaxy classification. It was performed for all objects brighter then $m_{3}+3$ classified by the FOCAS package as galaxies, $m_{3}$ is the magnitude of the third brightest galaxy.

The sample of galaxy clusters analyzed by us is not statistically uniform. However, we ensure the reliability of star/galaxy separation, which allows us to state that each of our clusters contain galaxies only.

The basic parameters of the clusters included in the present study are given in Table 1 . The first column contains the cluster name, Cols. 2 and 3 give the right ascension and declination (1950) of the cluster center, and Col. 3 shows the cluster redshift from Struble \& Rood (1987). In our analysis, we took into account all galaxies within the radius of $R=1.5 \mathrm{Mpc}$ from the cluster center and with a magnitude range from $m_{3}$ to $m_{3}+3$, where $m_{3}$ is the magnitude of the third brightest galaxy.

The completeness of each galaxy cluster sample has been checked using the $\log (N)-m$ dependence. In all cases, the completeness covered a range of at least 3 mag. For 29 of these clusters Trévese et al. (1996) studied the luminosity function. As seen from their Table 1 and Fig. 1, the sampling was done for $M^{\star}+2$ mag. For clusters A157, A779, A2028 and A2056 the magnitude limit was brighter than $m_{3}+3$. This can be related to the fact that the automatic star/galaxy classification methods break down for weak images of galaxies on the photographic plates, or to the low population of galaxies in the clusters.

\section{Wavelet analysis}

The detection of structure in the regions studied was achieved by a wavelet analysis (Escalera et al. 1994). The wavelet technique is a convolution on a grid of $N \times N$ pixels between the signal $s(r)$ (in our case, the angular positions of galaxies) and the analyzing wavelet function $g(r, a)$. Following Escalera \& Mazure (1992), we use the two-dimensional radial function called the Mexican Hat, given by the formula:

$g(r, a)=\left(2-\frac{r^{2}}{a^{2}}\right) \exp \left(-\frac{r^{2}}{2 a^{2}}\right)$,

where $r$ is the distance between the position of a galaxy and point $(x, y)$ where the wavelet coefficient is calculated, and $a$ is a scale length for the wavelet in order to form the corresponding set of wavelet coefficients. As a result of the convolution, the signal is transformed into a set of wavelet coefficients given by:

$w(r, a)=g(r, a) \otimes s(r)$.

Each pixel in the grid then has a corresponding wavelet coefficient. Using a set of different scales, a structure is detected only when its characteristic size is of the order of the scale applied. Following Daubechies (1990), the factor of $\sqrt{2}$ from 
one scale to another ensures correct sampling in the case of the Mexican Hat. When analyzed with the largest scale, the field will produce a wavelet image showing a single central structure. If the scale decreases, the central structure either remains unchanged or splits into substructures. In this way, we can detect all structures present in the map, irrespective of their location or size. For the analysis presented here, the discrete wavelet was computed on a grid of $256 \times 256$ pixels for seven scales increasing from $a=8$ to 64 (in pixel units), namely $8,11,16,22,32,45,64$, corresponding to $94,129,188$, $258,375,527,750 \mathrm{kpc}$. In order to avoid any edge effects, areas larger than the cluster itself were analyzed.

We modeled the significance of the substructure detected using Monte Carlo simulations. For each cluster and each scale $a$, a wavelet analysis was carried out on a set of 1000 randomly generated distributions of galaxies containing the same number of points as in the actual fields.

We assume that a substructure is real if the probability that the detected substructure is due to random fluctuations is less than $1 \%$. Furthermore, for each scale $a$ only substructures with more than 4 galaxy members in a circle of radius $a$ are considered.

In this way, we detected all structures in the field of the galaxy clusters studied here. Following Escalera et al. (1994), we classified the structures morphologically according to three categories: unimodal, bimodal and complex.

Some of the detected substructures are due to projection effects. However, the application of 1000 simulations for each scale $a$ allows one to estimate the probability of the existence of the detected substructure in the random distribution of galaxies, and it is small. Theoretically, subclustering existing in 3D data should be also detected in 2D data. However, it may happen that two subgroups are located along the line-of-sight, thus appearing in projection as one group. The projection effect is of the same importance for clusters with and without 3D substructures. Therefore, for the statistical investigations of the frequency of substructure occurrence this is not a very important factor.

\section{Results}

The results of our wavelet analysis applied to the present sample of galaxy clusters are given in Table 3. Column 1 gives the cluster name, column 2 identifies the structure $(\mathrm{M}-$ main cluster; A - dominant central structure; B, C - substructures), Col. 3 shows the wavelet scales $a$ at which the structures were detected, with values in pixel units for a map of $256 \times 256$ pixels, Col. 4 gives the number of galaxies belonging to the relevant structures, and Cols. 5 and 6 show coordinates for the center of the detected structure. Column 7 gives cluster morphology ( $U$ - unimodal, $S_{f}$ - substructures present in the field of cluster, and $\mathrm{S}_{\mathrm{c}}-$ substructures in the cluster core).

We assume that a cluster has substructures in the core $\left(\mathrm{S}_{\mathrm{c}}\right)$ if the distance between the center of the detected substructure and the center of the main cluster is smaller than $375 \mathrm{kpc}$. If it is located at a greater distance, the cluster has substructures in the field $\left(\mathrm{S}_{\mathrm{f}}\right)$.

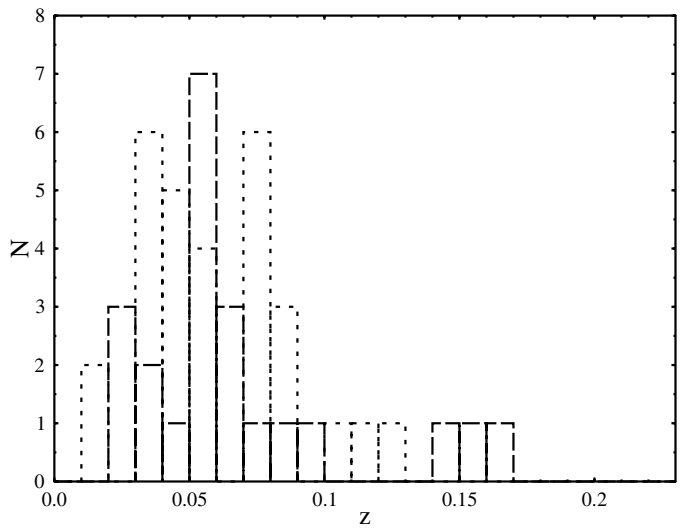

Fig. 1. The distribution of the galaxy clusters with substructures versus redshifts for the morphological type $S_{c}$ (solid line) and $S_{f}$ (dotted line).

Using the classification scheme for clusters of galaxies developed by Rood \& Sastry (1971) and by Bautz \& Morgan (1970) the distribution of the clusters having substructures for different morphological types is presented in Table 2. Excluding results for types $\mathrm{L}$ and $\mathrm{B}$, which are poorly populated, the $\chi^{2}$ test shows no correlation between the existence of subclustering and the type of galaxy cluster at the significance level $\alpha=0.05$. Also the $\mathrm{S}_{\mathrm{f}}$ and $\mathrm{S}_{\mathrm{c}}$ substructures do not depend on the BM and RS cluster morphological types.

We tested if the dependence $S_{c}$ versus redshift and $S_{f}$ versus redshift is the same (Fig. 1). Using the Kolmogorov-Smirnov test we did not find any statistically significant difference at the level $\alpha=0.05$ between the distributions. The test carried out for bins of $0.005,0.010$ and 0.015 lead to the same conclusion.

In Figs. 3-18 we show only 62 of the clusters with detected substructures. The plots show the distribution of galaxies and the wavelet images on a grid of $256 \times 256$ pixels for six wavelet scales $a=129,188,258,375,527,750 \mathrm{kpc}$. From these plots it is possible to identify unimodal or bimodal clusters, and substructures. In general, the central A - structure can still be seen at small scales, although the corresponding physical structure is clearly defined at the largest scales only.

Thus, the wavelet analysis shows statistically significant substructures in $34 \%$ of the clusters in our sample. According to the frequency of clusters with statistically significant substructures, the results presented in this paper are consistent with those of other authors, based on 2D or 3D data.

\section{Discussion}

Below we present some comments on substructures in particular clusters.

A76 - At the scales of $a=375 \mathrm{kpc}$ and $a=258 \mathrm{kpc}$, this cluster has two galaxy groups in the cluster field.

A117 - At the scale of $a=188 \mathrm{kpc}$ substructures are located in the central region of the cluster.

A121 - Substructures are identified at scales $a=375 \mathrm{kpc}$ and $a=258 \mathrm{kpc}$.

A133 - In this bimodal cluster, small subclusters occur at the scale of $a=188 \mathrm{kpc}$. 
A140 - At the scale $a=188 \mathrm{kpc}$, this cluster has one group of galaxies.

A151 - Two significant substructures at the scale $a=$ $375 \mathrm{kpc}$.

A261 - This is bimodal cluster at scale of $a=527 \mathrm{kpc}$.

A358 - Substructures exists in the central region of the cluster at the scale of $a=129 \mathrm{kpc}$.

A415 - Two significant substructures occur at the scales $a=$ $188 \mathrm{kpc}$ and $a=258 \mathrm{kpc}$.

A426 - The substructures are located in the field of the cluster at the all scales.

A496 - The significant substructures occur at the all scales in the field of the cluster.

A514 - This is an elongated cluster with substructures at the scales of $a=375 \mathrm{kpc}$ and $a=188 \mathrm{kpc}$.

A527 - One group of galaxies located at the external part of the cluster.

A539 - At the scales $a=258 \mathrm{kpc}$ and $a=188 \mathrm{kpc}$ small groups of galaxies are located at the central part of the cluster.

A548 - In this bimodal cluster, substructures exists at the scales $a=188 \mathrm{kpc}$ and $a=94 \mathrm{kpc}$.

A671 - This cluster has two significant substructures in the cluster center at the scale of $a=129 \mathrm{kpc}$. Each of them has 8 galaxy members.

A754 - At the scales of $a=188 \mathrm{kpc}$ and $a=129 \mathrm{kpc}$ there are three substructures.

A786 - Only one substructure at the scale of $a=375 \mathrm{kpc}$.

A924 - Two groups of galaxies in the central region of cluster, at the scale of $a=375 \mathrm{kpc}$.

A978 - In this symmetrical cluster two substructures exists at the scale of $a=258 \mathrm{kpc}$.

A1016 - At the scale $a=188 \mathrm{kpc}$, this cluster has significant substructures at the cluster center.

A1060 - There are six substructures at the scales $a=375 \mathrm{kpc}$ and $a=129 \mathrm{kpc}$.

A1187 - This bimodal cluster has two substructures at the scale of $a=527 \mathrm{kpc}$.

A1275 - Two significant substructures located symmetrically according cluster center.

A1292 - One substructure at the outer part of cluster, $a=$ $258 \mathrm{kpc}$.

A1367 - At the scale of $a=129 \mathrm{kpc}$ this cluster has two galaxy groups.

A1377 - Two significant substructures occur at the scales of $a=129 \mathrm{kpc}$ and $a=94 \mathrm{kpc}$.

A1401 - Bimodal cluster, substructures at the scale of $a=$ $527 \mathrm{kpc}$.

A1413 - This is an elongated cluster with two close groups of galaxies at the scale of $a=375 \mathrm{kpc}$.

A1631 - This cluster has substructures at the scale $a=$ $188 \mathrm{kpc}$ in the cluster center.

A1650 - This cluster has substructures in the cluster field.

A1651 - One group of galaxies at the scale of $a=375 \mathrm{kpc}$ in the field of the galaxies.

A1661 - This elongated cluster has one group of galaxies at the scale of $a=375 \mathrm{kpc}$.

A1736 - The substructures occur at the scales of $a=188 \mathrm{kpc}$.
A1809 - In this cluster the substructures are located in the central region at the scale $a=258 \mathrm{kpc}$.

A1831 - This cluster has two significant substructures in the cluster center at the scale of $a=188 \mathrm{kpc}$.

A1837 - The substructures are located in the field of the cluster.

A1904 - This cluster has one substructure in the field at the scale of $a=129 \mathrm{kpc}$.

A1913 - Statistical significant substructures exists at the scale $a=188 \mathrm{kpc}$.

A1983 - This is a bimodal cluster.

A2020 - This is a bimodal cluster.

A2022 - One group of galaxies at the scale of $a=129 \mathrm{kpc}$.

A2028 - In this elongated cluster, substructure exists at the scale of $a=258 \mathrm{kpc}$. It is located in the upper region of the field.

A2052 - The substructures at the scale $a=375 \mathrm{kpc}$ exists in the external region of the cluster.

A2065 - One group of galaxies in the external part of the cluster occurs at the scale of $a=258 \mathrm{kpc}$.

A2073 - In this almost regular cluster, the wavelet analysis identified one substructure in the core region, at the scale of $a=188 \mathrm{kpc}$.

A2124 - At the scale of $a=129 \mathrm{kpc}$, two small substructures are located in the central region of the cluster.

A2147 - In this cluster two groups of galaxies occurs at the scale of $a=375 \mathrm{kpc}$.

A2151 - This is a bimodal cluster.

A2152 - This bimodal cluster has substructure at the scale of $a=258 \mathrm{kpc}$.

A2199 - At the scales of $a=129 \mathrm{kpc}$ and $a=94 \mathrm{kpc}$ substructures occurs in the field of the cluster.

A2255 - This cluster has one significant substructure in the cluster center at the scale of $a=129 \mathrm{kpc}$.

A2312 - One small substructure in the center of the cluster, at the scale $a=188 \mathrm{kpc}$.

A2457 - This cluster has one group of galaxies in the center, at the scale $a=129 \mathrm{kpc}$.

A2593 - Only one small group of galaxies is present in the core region of the cluster, at the scale of $a=188 \mathrm{kpc}$.

A2877 - Only two small substructures occurs at the scale of $a=94 \mathrm{kpc}$.

A3128 - In this cluster the substructures exists at all the scales smaller than $a=375 \mathrm{kpc}$.

A3376 - Two groups of galaxies at the scales of $a=188 \mathrm{kpc}$ and $a=129 \mathrm{kpc}$.

A3395 - This is the bimodal cluster.

A3526 - This bimodal cluster has small substructures at the scales of $a=375 \mathrm{kpc}, a=258 \mathrm{kpc}$ and $a=188 \mathrm{kpc}$.

A3562 - In this cluster substructures occurs at the scales of $a=258 \mathrm{kpc}$ and $a=129 \mathrm{kpc}$.

A3667 - This cluster has significant substructures at the scales of $a=258 \mathrm{kpc}$ and $a=94 \mathrm{kpc}$.

\section{Conclusions}

The method based on a wavelet transform has been applied for substructure detection in the projected distributions of galaxies 

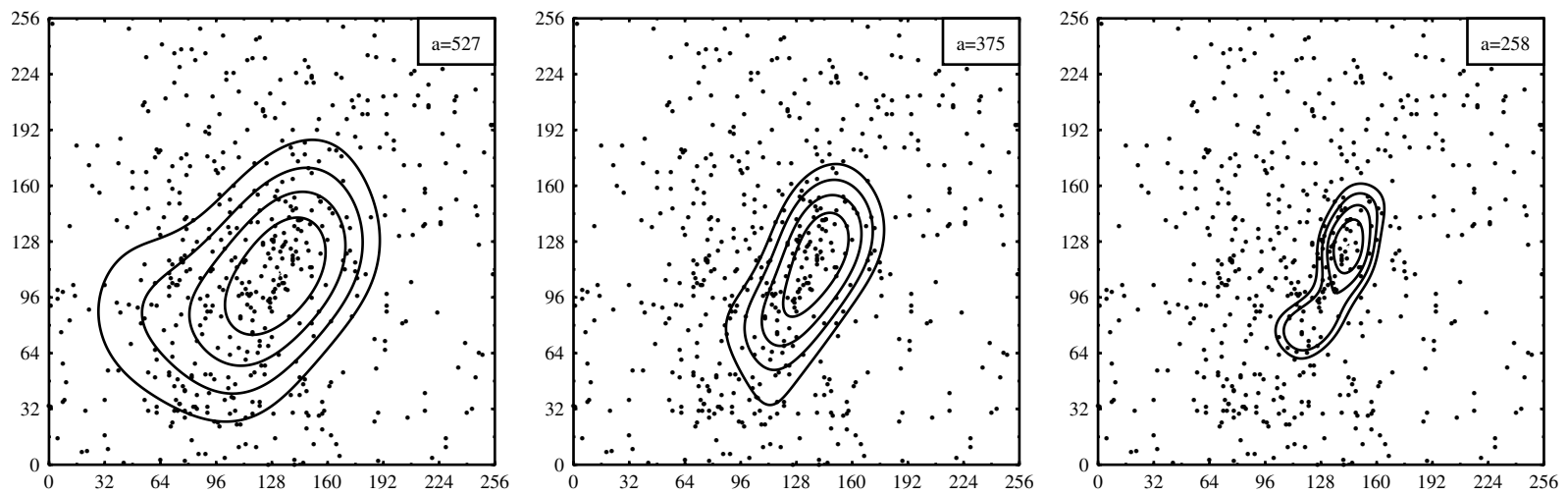

Fig. 2. Distribution of galaxies in A85 with the wavelet images for scales $a=527,375,258 \mathrm{kpc}$ presented in the upper right box (galaxies within magnitude range from $m_{3}$ to $\left.m_{3}+3\right)$.

in 183 Abell clusters. The significance of substructure detected has been calibrated using simulated distributions by means of Monte Carlo modeling, performing 1000 simulations for each cluster and each scale. Applying the Mexican Hat wavelet analysis to the projected distributions of galaxies in 183 clusters, we conclude that significant structure is present in 62 , that is $34 \%$ of the cases.

Our results indicate that a large fraction of clusters have substructures in their projected galaxy distributions. A comparison with other investigations based both on the projected and on the $3 \mathrm{D}$ data shows that, while in individual cases there are sometimes differences in finding substructures, the percentage of substructure occurrence is not greatly affected. We find no correlation between frequency of the clusters with subclustering and their morphological type.

Our results are in agreement with the Plionis (2001) investigation. He applied two different methods for substructure detection in 309 ACO clusters for which only about 180 have measured redshifts. In our studies all clusters have measured redshifts, so our samples are almost identically numerous.

The influence of magnitude limits on subclustering was examined by Flin \& Krywult (2000). This was done using symmetry and separation tests (West et al. 1988). The subclustering depends neither on the magnitude range of considered galaxies, which is $m_{3}+2, m_{3}+3$, and $m_{3}+4$, nor the possible errors in determination of the third brightest galaxy. They checked this for $m_{i}+3$, where $i=3 \ldots 10$.

This shows that the search for subclustering does not depend on the magnitude limit, when $m_{3}+3$ or $m_{3}+4$ is considered, but when this magnitude range is significantly enlarged, substructures could be observed.

We applied our method to published optical data (Slezak et al. 1997) of the very rich cluster Abell 85 (Fig. 2). The data were analysed in the same manner as ours. We took into account all galaxies within a radius of $R=1.5 \mathrm{Mpc}$ from the cluster centre. To check the influence of the magnitude limit on the detected substructures the analysis was carried out on two sets of catalogues. The first catalogue was magnitude limited at the level $m_{3}+3$, the second one at the level $m_{3}+4$.

The results of a wavelet analysis show no statistically significant substructures in neither case. Due to increases in the number of galaxies in this catalogues from 317 to
587, the wavelet analysis showed some substructures, but they are not statistically significant at our adopted probability level. However, when all Slezak et al. (1997) data covering a magnitude range much larger than $m_{3}+4$ were considered, subclustering was detected.

The influence of the cluster centre chosen has been analyzed for clusters using mean, median and ACO catalogue centres. In some cases were found a differences between subclustering but the difference are not statistically significant.

In some tests to detect substructures in galaxy clusters there is a strong dependence on the cluster centre choice. This is the case of the symmetry test, the Lee test and others. A detailed discussion is given using numerical simulations by Pinkey et al. (1996).

The wavelet analysis performs a convolution of the analysing function (e.g. Mexican Hat) with positions of galaxies in cluster regions. Contrary to previously mentioned tests this method do not use the predetermined cluster centre for calculation. Our earlier studies show that the choice of the cluster centre is an important factor influencing the frequency of substructure occurrence.

Our considerations also show that the percentage of subclustering using a wavelet analysis applied to 2D data is close to that obtained from 3D data.

In A496 the wavelet analysis shows one substructure located in the outer region of this cluster. Durret et al. (2000) find only small groups of galaxies located in the central region of the cluster, but no significant substructures. In Fig. 9 of their paper there is one group of galaxies located in the outer region of A496, which corresponds to substructure detected by us. This comparison shows that different methods of analysis detect different substructures.

The results presented in this paper are in agreement with Burgett et al. (2004). They applied four statistical tests of the substructure analysis to the 25 low-richness clusters from the 2ndFGRS catalogue of galaxy clusters. They conclude that substructures exist in $20 \%$ to $44 \%$ of clusters, depending on the method applied.

Acknowledgements. We thank the anonymous referee for useful comments which led to the improvement of the paper. 


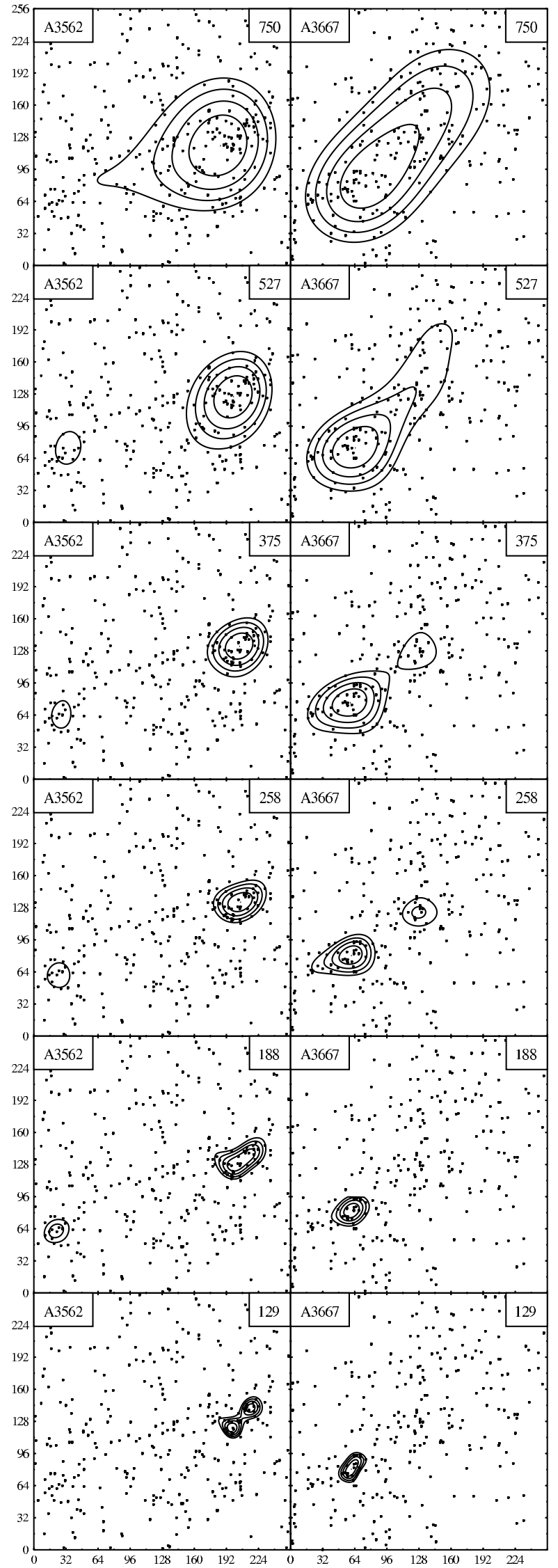

Fig. 18. Distribution of galaxies in A3562 and A3667 with wavelet images for scales $a=129,188,258,375,527,750 \mathrm{kpc}$ presented in the upper right box.

\section{References}

Abell, G. O., Corwin, H. G. Jr., \& Olowin, R. P. 1989, ApJS, 70, 1

Baier, F. W. 1979, Astron. Nachr., 300, 133

Baier, F. W. 1983, Astron. Nachr., 304, 211

Baier, F. W., \& Mai, W. 1977, Astron. Nachr., 298, 301

Baier, F. W., \& Mai, W. 1978, Astron. Nachr., 299, 69

Bautz, L. P., \& Morgan, W. W. 1970, ApJ, 162, L149

Bird, C. M. 1993, Ph.D. Thesis, University of Minnesota

Biviano, A., Katgert, P., Thomas, T., \& Adami, C. 2002, A\&A, 387, 8

Bothun, D. G., Aaroson, M., Schommer, B., et al. 1985, ApJS, 57, 423

Burgett, W. S., Vick, M. M., Davis, D. S., et al. 2004, MNRAS, 352, 605

Daubechies I. 1990, I.E.E.E. Trans. on Information Theory, 36, 961

Dressler, A., \& Shectman, S. A. 1988, AJ, 95, 985

Durret, F., Adami, C., Gerbal, D., \& Pislar, V. 2000 [arXiv:astro-ph/0002519]

Escalera, E., Biviano, A., Girardi, M., et al. 1994, AJ, 423, 539

Escalera, E., \& Mazure, A. 1992, ApJ, 388, 23

Flin, P., \& Krywult, J. 2000, ASP Conf. Ser., 200, 406

Flin, P., \& Krywult, J. 2002, ASP Conf. Ser., 268, 365

Flin, P., Trévese, D., Cirimele, G., \& Hickson, P. 1995, A\&AS, 110, 313

Flin, P., Krywult, J., Trévese, D., Cirimele, G., \& Hickson, P. 2000, A\&AS, 146, 373

Flin, P., Krywult, J., \& Biernacka, M. 2004 [arXiv:astro-ph/0404182]

Floor, S., Melott, A., \& Motl, P. [arXiv: astro-ph/0307539]

Geller, M. J., \& Beers, T. C. 1982, PASP, 94, 421

Gunn, J. E., \& Oke, J. B. 1975, ApJ, 195, 255

Hickson, P. 1977, ApJ, 217, 16

Hoessel, J. G., \& Schneider, D. P. 1985, AJ, 90, 1648

Hoessel, J. G., Gunn, J. E., \& Thuan, T. X. 1980, ApJ, 241, 486

Jarvis, J. F., \& Tyson, J. A. 1981, AJ, 86, 476

Kauffmann, G., \& White, S. D. M. 1993, MNRAS, 261, 921

Knebe, A., \& Muller, V. 2000, A\&A, 354, 761

Kolokotronis, V., Basilakos, S., Plonis, M., \& Georgantopoulos, I. 2002, MNRAS, 32, 49

Kriessler, J. R., \& Beers, T. C. 1997, AJ, 113, 80

Krywult, J. 1997, Kinematics and Physics of Celestial Bodies, 5, 36

Krywult, J., MacGillivray, H. T., \& Flin, P. 1999, A\&A, 351, 883

Murphy, H. P., Schild, R. E., \& Weeks, T. C. 1983, MNRAS, 202, 1127

Oemler, A. 1974, ApJ, 194, 1

Peterson, B. A. 1970, AJ, 75, 695

Pinkney, J., Roettiger, K., Burns, J. O., \& Bird, C. 1996, ApJS, 104, 1

Plionis, M. 2001 [arXiv: astro-ph/0110616]

Plionis, M. 2002, ApJ, 572, L67

Rahman, N., Shandarin, S. F., Motl, P. M., \& Melott, A. L. 2004 [arXiv: astro-ph/0405097]

Richstone, D., Loeb, A., \& Turner, E. L. 1992, ApJ, 393, 477

Rood, H. J., \& Sastry, G. N. 1971, PASP, 83, 313

Slezak, E., Durret, F., Guibert, J., \& Lobo, C. 1997 [arXiv: astro-ph/9710103]

Struble, M. F., \& Rood, H. J. 1987, ApJS, 63, 555

Schuecker, P., Boehringer, H., Reiprich, H. T., \& Feretti, L. 2001, A\&A, 378, 408

Thomas, P. A., Colberg., J. M., Couchman, H. M. P., et al. 1998, MNRAS, 296, 1061

Sandage, A., \& Perlmuter, J. M. 1991, ApJ, 370, 455

Trévese, D., Flin, P., Migliori, L., Hickson, P., \& Pittella, G. 1992, A\&AS, 94, 327

Trévese, D., Cirimele, G., \& Apodia, B. 1996, A\&A, 315, 365

Trévese, D., Cirimele, G., Cenci, A., et al. 1997, A\&AS, 125, 459

West, M. J., \& Bothun, G. D. 1990, ApJ, 350, 36

West, M. J., Oemler, A., \& Dekel, A. 1988, ApJ, 327, 1 


\section{Online Material}


P. Flin and J. Krywult: Substructures in Abell clusters of galaxies, Online Material $p 2$

Table 1. List of studied clusters.

\begin{tabular}{|c|c|c|c|c|c|c|c|c|c|c|c|}
\hline Cluster & $\bar{\alpha}$ & $\bar{\delta}$ & $z$ & Cluster & $\bar{\alpha}$ & $\delta$ & $z$ & Cluster & $\bar{\alpha}$ & $\delta$ & 2 \\
\hline A14 & 0012.7 & -2410 & 0.0640 & A439 & 0327.5 & +2437 & 0.1063 & A1146 & 1058.9 & -2227 & 0.141 \\
\hline A 21 & 0017.9 & +2821 & 0.0948 & A449 & 0343.5 & +7502 & 0.0803 & 1185 & 1108.1 & +2857 & 0.0304 \\
\hline A 22 & 0018.2 & -2559 & 0.1432 & A465 & 0348.3 & +0609 & 0.0855 & A1187 & 1108.9 & +3951 & 0.0791 \\
\hline A76 & 0037.2 & +0630 & 0.0416 & A496 & 0431.3 & -1321 & 0.0320 & A1190 & 1109.0 & +4107 & 0.0794 \\
\hline A85 & 0039.1 & -0937 & 0.0518 & A500 & 0436.8 & -2212 & 0.0666 & A 1213 & 1113.8 & +2932 & 0.0468 \\
\hline A88 & 0040.4 & -2619 & 0.1086 & A505 & 0451.6 & +7956 & 0.0543 & A1216 & 1115.2 & -0412 & 0.0524 \\
\hline A104 & 0047.1 & +2415 & 0822 & A514 & 0445.5 & -2031 & 0.0731 & A1228 & 1118.8 & +3436 & 0.0350 \\
\hline A114 & 0051.2 & -2157 & 0.0566 & A526 & 0457.2 & +0522 & 0.0541 & A1275 & 1127.3 & +3657 & .0603 \\
\hline A115 & 0053.3 & +2603 & 1971 & A527 & 0504.0 & +7338 & 0.0794 & A1292 & 1129.1 & +3606 & 0.2319 \\
\hline A117 & 0053.5 & -1018 & & A537 & 0518.3 & +7350 & 0.168 & 299 & 1129.7 & +3415 & 0.2247 \\
\hline A119 & 0053.8 & -0132 & 0.0440 & A539 & 0513.9 & +0624 & 0.2050 & A1314 & 1132.1 & +4919 & 0.0341 \\
\hline A121 & 0055.0 & -0717 & .1048 & A548 & 0545.0 & -2538 & 0.041 & A1334 & 1136.3 & -0402 & 0.0555 \\
\hline A133 & 0100.2 & -2204 & 0.0604 & A564 & 0655.2 & +6953 & 0.0779 & A1346 & 1138.6 & +0558 & 0.0970 \\
\hline A 140 & 0102.1 & -2414 & 0.152 & A566 & 0659.8 & +6322 & 0.0984 & A1364 & 1141.1 & -0129 & 0.1070 \\
\hline A147 & 0105.6 & +0154 & 0.0438 & A576 & 0717.3 & +5550 & 0.0381 & A1365 & 1141.8 & +3111 & 0.0763 \\
\hline A151 & 0106.4 & -1541 & 0.0526 & A592 & 0739.9 & +0929 & 0.0624 & A1367 & 1141.9 & +2007 & 0.0215 \\
\hline A157 & 0108.6 & -1440 & 0.119 & A637 & 0811.4 & +4832 & 0.168 & A1377 & 1144.3 & +5601 & 0.0514 \\
\hline A166 & 0112.1 & -1632 & 0.1156 & A644 & 0815.0 & -0726 & 0.0704 & A1401 & 1149.5 & +3733 & 0.1648 \\
\hline A168 & 0112.6 & -0001 & & A646 & 0818.6 & +4716 & 0.1303 & A1412 & 1153.1 & +7345 & 0.0839 \\
\hline A189 & 0121.1 & +0123 & 0.0335 & A649 & 0820.6 & +4902 & 0.150 & A1413 & 1152.8 & +2339 & 0.1427 \\
\hline & & & & & & +4717 & & & & +0337 & 339 \\
\hline A194 & 0123.0 & -0146 & 0.0178 & A671 & 0825.4 & +3035 & 0.0494 & A1474 & 1205.4 & +1514 & 0.0791 \\
\hline A195 & 012 & +1855 & & & 0832.1 & +3702 & 0.0 & 524 & 12 & +0807 & 0.1369 \\
\hline A 260 & 0149.0 & +3255 & 0.0348 & A754 & 0906.4 & -0926 & 0.0528 & A1541 & 1224.9 & +0907 & 0.0892 \\
\hline A261 & 0148.9 & -0229 & 0.0467 & A757 & 0909.4 & +4755 & 0.0515 & A1570 & 1233.9 & +2005 & 0.180 \\
\hline A262 & 0149.9 & +3554 & & A779 & 0916.8 & +3359 & 0.0226 & A1589 & 1239.1 & +1852 & 0.0718 \\
\hline A274 & 0152.2 & -0631 & 0.1289 & A786 & 0923.7 & +7501 & 0.1241 & A1630 & 1249.2 & +0450 & 0.0649 \\
\hline A277 & 0153.3 & -0737 & & A924 & 1004.1 & +3554 & 0.0989 & A1631 & 1250.2 & -1510 & 0.0508 \\
\hline A278 & 0154.4 & +3159 & 0.0896 & A978 & 1018.0 & -0616 & 0.0527 & A1644 & 1254.6 & -1705 & 0.0449 \\
\hline A279 & 0153.8 & +0049 & 0.0797 & A979 & 1017.9 & -0738 & 0.055 & A1650 & 1256.2 & -0129 & 0.0845 \\
\hline A 358 & 0227.8 & -1325 & 0.0576 & A1016 & 1024.4 & +1114 & 0.0321 & A1651 & 1256.8 & -0355 & 0.0825 \\
\hline A389 & 0249.1 & -2507 & 0.1160 & & 1029.2 & +4028 & 0.0799 & A1661 & 1259.4 & +2921 & 0.1671 \\
\hline A399 & 0255.2 & +1249 & 0.0715 & A1060 & 1034.5 & -2716 & 0.0114 & A1675 & 1302.9 & +3449 & 0.184 \\
\hline A401 & 0256.2 & +1323 & 0.0748 & & 1042.0 & +3550 & & A1689 & 1309.0 & -0106 & 0.181 \\
\hline A407 & 0258.6 & +3538 & 0.0470 & A1126 & 1051.3 & +1707 & 0.0852 & A1691 & 1309.1 & +3928 & 0.0722 \\
\hline A415 & 0304.4 & -1214 & 0.0788 & A1132 & 1055.3 & +5703 & 0.1363 & A1700 & 1312.3 & +2859 & 0.142 \\
\hline A426 & 0315.3 & +4120 & 0.0183 & A1139 & 1055.5 & +0146 & 0.0383 & A1736 & 1324.1 & -2651 & 0.035 \\
\hline
\end{tabular}


P. Flin and J. Krywult: Substructures in Abell clusters of galaxies, Online Material $p 3$

Table 1. continued.

\begin{tabular}{|c|c|c|c|c|c|c|c|c|c|c|c|}
\hline Cluster & $\overline{\alpha \alpha}$ & $\bar{\delta}$ & $z$ & Cluster & $\bar{\alpha}$ & $\overline{\delta \delta}$ & $z$ & Cluster & $\bar{\alpha}$ & $\bar{\delta}$ & $z$ \\
\hline A1749 & 327.3 & 3753 & & A2073 & 523.6 & +2835 & 0.120 & A2457 & 2233.2 & +0113 & 0.0597 \\
\hline A1774 & 39.0 & 016 & 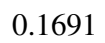 & 089 & 30.6 & +2811 & 743 & 93 & 2322.0 & -1422 & 0433 \\
\hline 775 & 39.6 & 637 & & 992 & .3 & 19 & 69 & 97 & 2322.7 & -1223 & 0852 \\
\hline A1795 & 1346.7 & +2650 & 6 & 2096 & 1533.5 & +3730 & 134 & 2634 & 2335.8 & +2645 & .0312 \\
\hline 309 & 50.8 & 524 & & 107 & 37.6 & +2156 & 421 & 557 & 2342.3 & +0852 & .0414 \\
\hline A1831 & 1356.9 & +2814 & 0.0733 & A2124 & 1543.1 & +3613 & 0.0654 & 2665 & 2348.2 & +0550 & 0.0556 \\
\hline A1837 & 1359.1 & -1055 & 376 & 2142 & 1556.2 & +2722 & 0.0899 & 2666 & 2348.4 & +2652 & 0.0265 \\
\hline A1904 & 1420.3 & +4847 & 8 & A 2147 & 1600.0 & +1602 & 0.0356 & A 2670 & 2351.6 & -1041 & 0.0745 \\
\hline A1913 & 24.5 & +1654 & 533 & 2151 & 1603.0 & +1753 & 0.0371 & A 2700 & 0001.3 & +0147 & 0.0978 \\
\hline & & & & & & & & & & & \\
\hline A1942 & 36.1 & +0353 & 0.224 & A 2175 & 1618.4 & +3002 & 0.0978 & A 2721 & 0003.6 & -3500 & 0.114 \\
\hline & & & & 197 & 1626.5 & +4101 & 0303 & 2877 & 0107.6 & -4610 & 0241 \\
\hline A1991 & 1452.2 & +1850 & 0586 & A2199 & 1626.9 & +3938 & 0.0303 & A 3128 & 0328.8 & -5244 & 0.0554 \\
\hline A2020 & 1501.3 & +0807 & 578 & A2210 & 1632.3 & +0535 & 0.1465 & A3266 & 0430.5 & -6135 & 0.0594 \\
\hline A2022 & 1502.2 & +2837 & 1 & A 2255 & 1712.2 & +6409 & 0.0800 & A3376 & 0559.1 & -4003 & 0.0455 \\
\hline A2028 & 07.1 & +0743 & 0772 & 256 & 1706.6 & +7847 & 0.0601 & A3391 & 0625.2 & -5339 & 0.0531 \\
\hline A2029 & 1508.5 & +0557 & 0.0767 & A2311 & 1850.2 & +7019 & 0.0675 & A3395 & 0626.5 & -5422 & 0.0498 \\
\hline A2040 & 1510.3 & +0737 & 0.0456 & A2312 & 1853.8 & +6818 & 0.0590 & A3526 & 1246.1 & -4102 & 0.0110 \\
\hline A2048 & 1512.8 & +0434 & & A2315 & 1901.2 & +6953 & 0.0822 & A3558 & 1325.1 & -3114 & 0.0482 \\
\hline A2052 & 1514.3 & +0711 & 0.0348 & A2319 & 1919.2 & +4352 & 0.0564 & A3562 & 1330.7 & -3125 & 0.0499 \\
\hline & & & & & & -0 & & 3667 & & -5658 & 0.0530 \\
\hline A2056 & 1517.1 & +2827 & 0.0763 & A2384 & 2149.5 & -1947 & 0.2384 & A3716 & 2047.9 & -5254 & 0.0456 \\
\hline A2063 & 1520.6 & +0849 & & & 2154.9 & -0802 & & A3888 & 2231.5 & -3759 & 0.1680 \\
\hline A2065 & 1520.6 & +2754 & 0.0721 & A2410 & 2159.4 & -1008 & 0.0806 & A4038 & 2345.1 & -2825 & 0.0283 \\
\hline
\end{tabular}


P. Flin and J. Krywult: Substructures in Abell clusters of galaxies, Online Material p 4

Table 2. The frequency of the galaxy clusters with significant substructures versus the morphological type.

\begin{tabular}{cccc}
\hline \hline Morphology & $\begin{array}{c}\text { Total } \\
\text { number }\end{array}$ & $\begin{array}{c}\text { Clusters with } \\
\text { substructures }\end{array}$ & Frequency \\
\hline cD & 47 & 13 & 28 \\
C & 35 & 12 & 34 \\
B & 12 & 1 & 8 \\
I & 38 & 7 & 18 \\
F & 31 & 14 & 45 \\
L & 12 & 5 & 42 \\
\hline
\end{tabular}

Table 3. Results of the wavelet analysis.

\begin{tabular}{|c|c|c|c|c|c|c|}
\hline 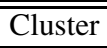 & Struct. & a-Scale & 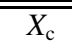 & $\overline{Y_{\mathrm{c}}}$ & 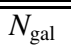 & Morph. \\
\hline A14 & $\mathrm{A}$ & 64 & 124 & 138 & 95 & $\mathrm{U}$ \\
\hline A 21 & A & 64 & 110 & 126 & 31 & $\mathrm{U}$ \\
\hline A22 & A & 64 & 130 & 106 & 89 & U \\
\hline \multirow{8}{*}{ A76 } & A & 64 & 121 & 134 & 43 & $S_{f}$ \\
\hline & & 45 & 107 & 139 & 24 & \\
\hline & B & 32 & 94 & 153 & 14 & \\
\hline & & & 156 & 120 & 13 & \\
\hline & $\mathrm{C}$ & 22 & 155 & 120 & 10 & \\
\hline & & & 95 & 177 & 10 & \\
\hline & & 16 & 152 & 118 & 6 & \\
\hline & & 11 & 91 & 119 & 5 & \\
\hline A85 & A & 64 & 109 & 117 & 119 & U \\
\hline A88 & A & 64 & 95 & 129 & 22 & $\mathrm{U}$ \\
\hline A104 & A & 64 & 110 & 109 & 62 & $\mathrm{U}$ \\
\hline A114 & A & 64 & 137 & 106 & 52 & $\mathrm{U}$ \\
\hline A115 & A & 64 & 109 & 184 & 59 & $\mathrm{U}$ \\
\hline \multirow[t]{8}{*}{ A117 } & A & 64 & 105 & 154 & 37 & $\mathrm{~S}_{\mathrm{c}}$ \\
\hline & & 45 & 90 & 158 & 21 & \\
\hline & & 32 & 79 & 161 & 13 & \\
\hline & & 22 & 79 & 162 & 10 & \\
\hline & B & 16 & 78 & 162 & 9 & \\
\hline & $\mathrm{C}$ & & 158 & 114 & 6 & \\
\hline & & 11 & 78 & 162 & 9 & \\
\hline & & 8 & 78 & 163 & 8 & \\
\hline A119 & A & 64 & 129 & 139 & 64 & $\mathrm{U}$ \\
\hline \multirow[t]{8}{*}{ A121 } & A & 64 & 127 & 123 & 53 & $\mathrm{~S}_{\mathrm{f}}$ \\
\hline & & 45 & 39 & 194 & 33 & \\
\hline & B & 32 & 36 & 197 & 19 & \\
\hline & $\mathrm{C}$ & 22 & 192 & 131 & 12 & \\
\hline & & & 33 & 198 & 13 & \\
\hline & & 16 & 192 & 131 & 12 & \\
\hline & & 11 & 191 & 131 & 8 & \\
\hline & & 8 & 190 & 130 & 5 & \\
\hline \multirow[t]{12}{*}{ A133 } & A & 64 & 121 & 135 & 81 & $\mathrm{~B}, \mathrm{~S}_{\mathrm{c}}$ \\
\hline & A1 & 45 & 111 & 138 & 55 & \\
\hline & $\mathrm{A} 2$ & & 213 & 39 & 46 & \\
\hline & & 32 & 107 & 138 & 42 & \\
\hline & & & 216 & 36 & 28 & \\
\hline & & 22 & 97 & 149 & 25 & \\
\hline & & & 123 & 123 & 20 & \\
\hline & & & 223 & 38 & 15 & \\
\hline & B1 & 16 & 127 & 121 & 18 & \\
\hline & B2 & & 95 & 155 & 16 & \\
\hline & & 11 & 126 & 122 & 10 & \\
\hline & & 8 & 126 & 124 & 7 & \\
\hline \multirow[t]{7}{*}{ A140 } & A & 64 & 106 & 109 & 76 & $\mathrm{~S}_{\mathrm{c}}$ \\
\hline & & 45 & 100 & 109 & 47 & \\
\hline & & 32 & 92 & 108 & 31 & \\
\hline & & 22 & 82 & 100 & 16 & \\
\hline & B & 16 & 79 & 97 & 13 & \\
\hline & & 11 & 78 & 97 & 9 & \\
\hline & & 8 & 79 & 98 & 6 & \\
\hline A147 & A & 64 & 182 & 119 & 43 & $\mathrm{U}$ \\
\hline \multirow[t]{6}{*}{ A151 } & A & 64 & 111 & 118 & 48 & $S_{f}$ \\
\hline & & 45 & 110 & 128 & 35 & \\
\hline & B & 32 & 122 & 127 & 23 & \\
\hline & $\mathrm{C}$ & & 125 & 26 & 17 & \\
\hline & & 22 & 127 & 128 & 14 & \\
\hline & & & 119 & 29 & 12 & \\
\hline
\end{tabular}


P. Flin and J. Krywult: Substructures in Abell clusters of galaxies, Online Material p 5

Table 3. continued.

\begin{tabular}{|c|c|c|c|c|c|c|}
\hline Cluster & Struct. & $a$-Scale & $\overline{X_{\mathrm{c}}}$ & $Y_{\mathrm{c}}$ & $N_{\text {gal }}$ & Morph. \\
\hline & & 16 & 130 & 131 & 11 & \\
\hline & & & 119 & 31 & 10 & \\
\hline & & 11 & 130 & 131 & 9 & \\
\hline & & & 119 & 32 & 8 & \\
\hline & & 8 & 132 & 132 & 6 & \\
\hline & & & 122 & 34 & 5 & \\
\hline A157 & A & 64 & 181 & 112 & 42 & $\mathrm{U}$ \\
\hline A166 & A & 64 & 135 & 141 & 27 & $\mathrm{U}$ \\
\hline A168 & A & 45 & 128 & 121 & 34 & $\mathrm{U}$ \\
\hline A189 & A & 64 & 44 & 191 & 25 & $\mathrm{U}$ \\
\hline A193 & A & 64 & 129 & 117 & 61 & $\mathrm{U}$ \\
\hline A194 & A & 64 & 117 & 119 & 49 & $\mathrm{U}$ \\
\hline A195 & A & 64 & 162 & 136 & 37 & $\mathrm{U}$ \\
\hline A260 & A & 64 & 134 & 88 & 30 & $\mathrm{U}$ \\
\hline \multirow[t]{8}{*}{ A261 } & A & 64 & 133 & 187 & 45 & B \\
\hline & A1 & 45 & 132 & 216 & 21 & \\
\hline & $\mathrm{A} 2$ & & 127 & 153 & 24 & \\
\hline & & 32 & 126 & 232 & 15 & \\
\hline & & 22 & 126 & 129 & 11 & \\
\hline & & 16 & 126 & 127 & 10 & \\
\hline & & 11 & 126 & 127 & 9 & \\
\hline & & 8 & 126 & 127 & 8 & \\
\hline A262 & A & 64 & 178 & 136 & 26 & $\mathrm{U}$ \\
\hline A274 & A & 64 & 105 & 130 & 29 & $\mathrm{U}$ \\
\hline A277 & A & 64 & 133 & 115 & 29 & $\mathrm{U}$ \\
\hline A278 & A & 64 & 180 & 103 & 60 & $\mathrm{U}$ \\
\hline A279 & A & 64 & 134 & 127 & 69 & $\mathrm{U}$ \\
\hline \multirow[t]{8}{*}{ A358 } & A & 64 & 117 & 118 & 50 & $\mathrm{~S}_{\mathrm{c}}$ \\
\hline & & 45 & 123 & 116 & 41 & \\
\hline & & 32 & 126 & 118 & 27 & \\
\hline & & 22 & 131 & 125 & 18 & \\
\hline & & 16 & 132 & 128 & 15 & \\
\hline & B & 11 & 131 & 130 & 13 & \\
\hline & $\mathrm{C}$ & & 98 & 106 & 8 & \\
\hline & & 8 & 131 & 130 & 7 & \\
\hline A389 & A & 64 & 73 & 152 & 34 & $\mathrm{U}$ \\
\hline A399 & A & 64 & 140 & 151 & 37 & $\mathrm{U}$ \\
\hline A401 & A & 64 & 130 & 119 & 39 & $\mathrm{U}$ \\
\hline A407 & A & 64 & 108 & 165 & 38 & $\mathrm{U}$ \\
\hline \multirow[t]{3}{*}{ A415 } & A & 64 & 95 & 150 & 28 & $S_{\mathrm{f}}$ \\
\hline & B & 22 & 69 & 147 & 10 & \\
\hline & $\mathrm{C}$ & 16 & 166 & 59 & 6 & \\
\hline \multirow[t]{17}{*}{ A426 } & A & 64 & 153 & 119 & 330 & $S_{\mathrm{f}}$ \\
\hline & & 45 & 154 & 118 & 243 & \\
\hline & & 32 & 154 & 119 & 184 & \\
\hline & & 22 & 153 & 120 & 138 & \\
\hline & B & & 143 & 241 & 30 & \\
\hline & & 16 & 153 & 121 & 113 & \\
\hline & & & 143 & 242 & 28 & \\
\hline & $\mathrm{C}$ & & 74 & 62 & 20 & \\
\hline & $\mathrm{D}$ & & 18 & 16 & 13 & \\
\hline & E & & 246 & 188 & 15 & \\
\hline & $\mathrm{F}$ & & 239 & 101 & 10 & \\
\hline & G & & 56 & 157 & 15 & \\
\hline & $\mathrm{H}$ & & 101 & 178 & 16 & \\
\hline & & 11 & 152 & 121 & 93 & \\
\hline & & & 144 & 242 & 24 & \\
\hline & & & 247 & 188 & 11 & \\
\hline & I & & 124 & 65 & 11 & \\
\hline
\end{tabular}

Table 3. continued.

\begin{tabular}{|c|c|c|c|c|c|c|}
\hline Cluster & Struct. & $a$-Scale & $X_{\mathrm{c}}$ & $Y_{\mathrm{c}}$ & $N_{\text {gal }}$ & Morph. \\
\hline & & & 20 & 14 & 8 & \\
\hline & & & 79 & 64 & 10 & \\
\hline & & & 61 & 157 & 9 & \\
\hline & & & 240 & 99 & 7 & \\
\hline & $\mathrm{J}$ & & 191 & 112 & 14 & \\
\hline & \multirow[t]{6}{*}{ K } & & 77 & 5 & 6 & \\
\hline & & \multirow[t]{5}{*}{8} & 152 & 121 & 83 & \\
\hline & & & 143 & 242 & 14 & \\
\hline & & & 190 & 111 & 9 & \\
\hline & & & 123 & 65 & 8 & \\
\hline & & & 79 & 6 & 5 & \\
\hline & \multirow[t]{5}{*}{$\mathrm{L}$} & & 170 & 183 & 6 & \\
\hline & & & 248 & 189 & 7 & \\
\hline & & & 239 & 98 & 7 & \\
\hline & & & 106 & 149 & 9 & \\
\hline & & & 165 & 88 & 9 & \\
\hline A439 & A & 64 & 112 & 142 & 50 & $\mathrm{U}$ \\
\hline A449 & A & 64 & 147 & 109 & 35 & $\mathrm{U}$ \\
\hline A465 & A & 64 & 134 & 133 & 28 & U \\
\hline \multirow[t]{13}{*}{ A496 } & A & 64 & 146 & 141 & 178 & $\mathrm{~S}_{\mathrm{f}}$ \\
\hline & B & 45 & 131 & 124 & 115 & \\
\hline & \multirow[t]{11}{*}{$\mathrm{C}$} & & 201 & 221 & 84 & \\
\hline & & 32 & 129 & 124 & 86 & \\
\hline & & & 200 & 228 & 57 & \\
\hline & & 22 & 131 & 124 & 53 & \\
\hline & & & 199 & 234 & 35 & \\
\hline & & 16 & 129 & 124 & 32 & \\
\hline & & & 201 & 240 & 21 & \\
\hline & & 11 & 201 & 242 & 18 & \\
\hline & & & 126 & 127 & 20 & \\
\hline & & 8 & 202 & 242 & 13 & \\
\hline & & & 127 & 127 & 14 & \\
\hline A500 & A & 64 & 144 & 125 & 36 & $\mathrm{U}$ \\
\hline A505 & A & 64 & 108 & 175 & 57 & $\mathrm{U}$ \\
\hline \multirow[t]{7}{*}{ A514 } & \multirow[t]{2}{*}{ A } & 64 & 80 & 102 & 50 & $\mathrm{~S}_{\mathrm{f}}$ \\
\hline & & 45 & 53 & 92 & 34 & \\
\hline & \multirow[t]{2}{*}{ B } & 32 & 46 & 82 & 20 & \\
\hline & & 22 & 41 & 74 & 13 & \\
\hline & \multirow[t]{3}{*}{$\mathrm{C}$} & 16 & 93 & 120 & 9 & \\
\hline & & & 42 & 72 & 10 & \\
\hline & & 11 & 170 & 159 & 6 & \\
\hline A526 & A & 64 & 97 & 141 & 38 & $\mathrm{U}$ \\
\hline \multirow[t]{5}{*}{ A527 } & A & 64 & 172 & 158 & 28 & $S_{\mathrm{f}}$ \\
\hline & \multirow[t]{4}{*}{ B } & 32 & 63 & 233 & 13 & \\
\hline & & 22 & 63 & 232 & 11 & \\
\hline & & 16 & 63 & 231 & 9 & \\
\hline & & 11 & 60 & 229 & 7 & \\
\hline A537 & A & 64 & 125 & 116 & 53 & $\mathrm{U}$ \\
\hline \multirow[t]{4}{*}{ A539 } & A & 64 & 130 & 118 & 96 & $S_{\mathrm{f}}$ \\
\hline & B & 22 & 20 & 18 & 10 & \\
\hline & $\mathrm{C}$ & 16 & 158 & 95 & 10 & \\
\hline & $\mathrm{D}$ & & 107 & 83 & 11 & \\
\hline \multirow[t]{7}{*}{ A548 } & A1 & 64 & 64 & 175 & 100 & $\mathrm{~B}, \mathrm{~S}_{\mathrm{f}}$ \\
\hline & \multirow[t]{6}{*}{$\mathrm{A} 2$} & & 197 & 107 & 90 & \\
\hline & & 45 & 48 & 178 & 57 & \\
\hline & & & 210 & 96 & 66 & \\
\hline & & 32 & 215 & 87 & 34 & \\
\hline & & & 38 & 182 & 33 & \\
\hline & & 22 & 35 & 186 & 26 & \\
\hline
\end{tabular}


P. Flin and J. Krywult: Substructures in Abell clusters of galaxies, Online Material $p 6$

Table 3. continued.

\begin{tabular}{|c|c|c|c|c|c|c|}
\hline Cluster & Struct. & $a-$ Scale & $X_{\mathrm{c}}$ & $Y_{\mathrm{c}}$ & $N_{\mathrm{gal}}$ & Morph. \\
\hline & \multirow{12}{*}{ B } & \multirow{3}{*}{16} & 214 & 79 & 23 & \\
\hline & & & 211 & 79 & 17 & \\
\hline & & & 37 & 185 & 17 & \\
\hline & & \multirow{7}{*}{11} & 184 & 133 & 15 & \\
\hline & & & 210 & 80 & 11 & \\
\hline & & & 184 & 134 & 13 & \\
\hline & & & 43 & 182 & 13 & \\
\hline & & & 103 & 227 & 7 & \\
\hline & & & 228 & 124 & 7 & \\
\hline & & & 22 & 189 & 9 & \\
\hline & & \multirow[t]{7}{*}{8} & 209 & 81 & 11 & \\
\hline & & & 186 & 135 & 7 & \\
\hline & C & & 95 & 85 & 6 & \\
\hline & \multirow[t]{4}{*}{ D } & & 136 & 146 & 5 & \\
\hline & & & 79 & 148 & 7 & \\
\hline & & & 44 & 185 & 6 & \\
\hline & & & 102 & 225 & 5 & \\
\hline A564 & A & 64 & 132 & 174 & 32 & $\mathrm{U}$ \\
\hline A566 & A & 64 & 123 & 139 & 34 & $\mathrm{U}$ \\
\hline A576 & A & 64 & 135 & 125 & 84 & $\mathrm{U}$ \\
\hline A592 & A & 64 & 86 & 109 & 29 & $\mathrm{U}$ \\
\hline A637 & A & 64 & 52 & 104 & 21 & $\mathrm{U}$ \\
\hline A644 & A & 64 & 136 & 116 & 166 & $\mathrm{U}$ \\
\hline A646 & A & 64 & 122 & 102 & 49 & $\mathrm{U}$ \\
\hline A649 & A & 64 & 46 & 78 & 17 & $\mathrm{U}$ \\
\hline A655 & A & 64 & 103 & 111 & 104 & $\mathrm{U}$ \\
\hline \multirow[t]{9}{*}{ A671 } & \multirow[t]{5}{*}{ A } & 64 & 125 & 125 & 69 & \multirow[t]{9}{*}{$S_{\mathrm{f}}$} \\
\hline & & 45 & 128 & 124 & 55 & \\
\hline & & 32 & 132 & 121 & 33 & \\
\hline & & 22 & 143 & 117 & 21 & \\
\hline & & 16 & 146 & 118 & 13 & \\
\hline & B1 & 11 & 146 & 120 & 8 & \\
\hline & \multirow[t]{3}{*}{ B2 } & \multirow{3}{*}{8} & 110 & 123 & 8 & \\
\hline & & & 146 & 120 & 7 & \\
\hline & & & 111 & 123 & 6 & \\
\hline A680 & A & 64 & 99 & 126 & 61 & $\mathrm{U}$ \\
\hline \multirow[t]{12}{*}{ A754 } & \multirow[t]{4}{*}{ A } & 64 & 108 & 123 & 102 & \multirow[t]{12}{*}{$\mathrm{S}_{\mathrm{f}}$} \\
\hline & & 45 & 107 & 127 & 75 & \\
\hline & & 32 & 113 & 130 & 54 & \\
\hline & & 22 & 118 & 131 & 34 & \\
\hline & B & 16 & 123 & 130 & 19 & \\
\hline & \multirow[t]{3}{*}{$\mathrm{C}$} & \multirow{3}{*}{11} & 84 & 114 & 17 & \\
\hline & & & 128 & 126 & 11 & \\
\hline & & & 81 & 111 & 9 & \\
\hline & D & & 161 & 169 & 6 & \\
\hline & & 8 & 128 & 125 & 10 & \\
\hline & & & 161 & 169 & 5 & \\
\hline & & & 211 & 104 & 5 & \\
\hline A757 & A & 64 & 127 & 135 & 36 & $\mathrm{U}$ \\
\hline A779 & A & 64 & 89 & 107 & 28 & $\mathrm{U}$ \\
\hline A786 & A & 64 & 167 & 117 & 54 & $S_{\mathrm{f}}$ \\
\hline & B & 32 & 35 & 103 & 24 & \\
\hline & & 22 & 39 & 99 & 15 & \\
\hline A924 & A & 64 & 142 & 129 & 86 & $\mathrm{~S}_{\mathrm{c}}$ \\
\hline & & 45 & 125 & 130 & 61 & \\
\hline & & 32 & 119 & 130 & 41 & \\
\hline & & 22 & 119 & 130 & 23 & \\
\hline & B1 & 16 & 120 & 139 & 16 & \\
\hline & B2 & & 117 & 119 & 15 & \\
\hline
\end{tabular}

Table 3. continued.

\begin{tabular}{|c|c|c|c|c|c|c|}
\hline Cluster & Struct. & $\overline{a-S c a l e}$ & $\overline{X_{\mathrm{c}}}$ & $\overline{Y_{\mathrm{c}}}$ & $\overline{N_{\mathrm{gal}}}$ & Morph. \\
\hline & & 11 & 82 & 245 & 7 & \\
\hline & & & 29 & 38 & 6 & \\
\hline & & & 121 & 143 & 8 & \\
\hline \multirow{9}{*}{ A978 } & A & 64 & 133 & 115 & 126 & $\mathrm{~S}_{\mathrm{c}}$ \\
\hline & & 45 & 130 & 116 & 87 & \\
\hline & & 32 & 126 & 110 & 46 & \\
\hline & B1 & 22 & 131 & 150 & 28 & \\
\hline & B2 & & 127 & 86 & 30 & \\
\hline & & 16 & 131 & 150 & 20 & \\
\hline & & & 126 & 82 & 21 & \\
\hline & & 11 & 131 & 149 & 14 & \\
\hline & & 8 & 132 & 148 & 8 & \\
\hline A979 & A & 64 & 127 & 163 & 52 & U \\
\hline \multirow[t]{11}{*}{ A1016 } & A & 64 & 129 & 96 & 59 & $\mathrm{~S}_{\mathrm{c}}$ \\
\hline & & 45 & 125 & 123 & 37 & \\
\hline & & 32 & 126 & 128 & 22 & \\
\hline & & 22 & 127 & 128 & 14 & \\
\hline & & & 112 & 19 & 12 & \\
\hline & & 16 & 119 & 137 & 10 & \\
\hline & & & 115 & 15 & 7 & \\
\hline & & & 153 & 120 & 10 & \\
\hline & B & 11 & 155 & 118 & 6 & \\
\hline & $\mathrm{C}$ & & 120 & 136 & 6 & \\
\hline & & 8 & 122 & 135 & 5 & \\
\hline A1035 & A & 64 & 129 & 138 & 77 & $\mathrm{U}$ \\
\hline \multirow[t]{22}{*}{ A1060 } & A & 64 & 135 & 135 & 356 & $\mathrm{~S}_{\mathrm{f}}$ \\
\hline & & 45 & 136 & 137 & 255 & \\
\hline & & 32 & 136 & 138 & 173 & \\
\hline & B & & 61 & 53 & 43 & \\
\hline & & 22 & 136 & 136 & 111 & \\
\hline & & & 76 & 60 & 24 & \\
\hline & & 16 & 136 & 135 & 82 & \\
\hline & & & 79 & 62 & 19 & \\
\hline & & 11 & 135 & 134 & 55 & \\
\hline & & & 81 & 63 & 15 & \\
\hline & $\mathrm{C}$ & & 179 & 152 & 18 & \\
\hline & D & & 160 & 85 & 14 & \\
\hline & E & & 102 & 165 & 16 & \\
\hline & $\mathrm{F}$ & & 35 & 39 & 9 & \\
\hline & G & & 121 & 95 & 16 & \\
\hline & & 8 & 135 & 133 & 31 & \\
\hline & & & 159 & 85 & 12 & \\
\hline & & & 180 & 153 & 11 & \\
\hline & & & 83 & 63 & 10 & \\
\hline & & & 35 & 39 & 9 & \\
\hline & & & 108 & 167 & 11 & \\
\hline & & & 91 & 160 & 9 & \\
\hline A1081 & A & 64 & 110 & 155 & 42 & $\mathrm{U}$ \\
\hline A1126 & A & 64 & 105 & 112 & 31 & $\mathrm{U}$ \\
\hline A1132 & A & 64 & 87 & 81 & 26 & $\mathrm{U}$ \\
\hline A1139 & A & 64 & 127 & 121 & 51 & $\mathrm{U}$ \\
\hline A1146 & A & 64 & 120 & 110 & 61 & $\mathrm{U}$ \\
\hline A1185 & A & 64 & 123 & 172 & 34 & $\mathrm{U}$ \\
\hline \multirow[t]{6}{*}{ A1187 } & A & 64 & 96 & 136 & 69 & B \\
\hline & A1 & 45 & 92 & 144 & 40 & \\
\hline & $\mathrm{A} 2$ & & 135 & 123 & 35 & \\
\hline & & 32 & 159 & 120 & 19 & \\
\hline & & & 90 & 168 & 22 & \\
\hline & & 22 & 89 & 168 & 17 & \\
\hline
\end{tabular}


P. Flin and J. Krywult: Substructures in Abell clusters of galaxies, Online Material $p 7$

Table 3. continued.

\begin{tabular}{|c|c|c|c|c|c|c|}
\hline Cluster & Struct. & $\overline{a-\text { Scale }}$ & 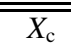 & $\overline{\overline{Y_{\mathrm{c}}}}$ & 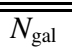 & Morph. \\
\hline & & & 155 & 119 & 15 & \\
\hline & & 16 & 87 & 168 & 13 & \\
\hline & & & 154 & 118 & 11 & \\
\hline & & 11 & 85 & 167 & 10 & \\
\hline & & & 152 & 115 & 8 & \\
\hline & & 8 & 84 & 168 & 6 & \\
\hline & & & 153 & 114 & 6 & \\
\hline A1190 & A & 64 & 134 & 133 & 62 & $\mathrm{U}$ \\
\hline A1213 & A & 64 & 144 & 100 & 37 & $\mathrm{U}$ \\
\hline A1216 & A & 64 & 128 & 116 & 41 & $\mathrm{U}$ \\
\hline A1228 & A & 64 & 102 & 117 & 54 & $\mathrm{U}$ \\
\hline \multirow[t]{11}{*}{ A1275 } & A & 64 & 132 & 128 & 42 & $\mathrm{~S}_{\mathrm{c}}$ \\
\hline & & 45 & 140 & 122 & 27 & \\
\hline & & 32 & 158 & 118 & 15 & \\
\hline & & & 109 & 124 & 16 & \\
\hline & B & 22 & 103 & 124 & 13 & \\
\hline & & & 164 & 117 & 11 & \\
\hline & & 16 & 106 & 121 & 9 & \\
\hline & $\mathrm{C}$ & & 160 & 119 & 9 & \\
\hline & & 11 & 107 & 119 & 8 & \\
\hline & & & 160 & 119 & 5 & \\
\hline & & 8 & 107 & 119 & 6 & \\
\hline \multirow[t]{4}{*}{ A1292 } & A & 64 & 136 & 139 & 58 & $S_{f}$ \\
\hline & & 45 & 136 & 131 & 31 & \\
\hline & B & 22 & 237 & 203 & 12 & \\
\hline & & & 127 & 135 & 13 & \\
\hline A1299 & A & 64 & 123 & 112 & 74 & $\mathrm{U}$ \\
\hline A1314 & A & 64 & 147 & 115 & 30 & $\mathrm{U}$ \\
\hline A1334 & A & 64 & 88 & 139 & 31 & $\mathrm{U}$ \\
\hline A1346 & A & 64 & 123 & 170 & 68 & $\mathrm{U}$ \\
\hline A1364 & A & 64 & 144 & 169 & 29 & $\mathrm{U}$ \\
\hline A1365 & A & 64 & 126 & 91 & 32 & $\mathrm{U}$ \\
\hline \multirow[t]{10}{*}{ A1367 } & A & 64 & 160 & 117 & 126 & $\mathrm{~S}_{\mathrm{c}}$ \\
\hline & & 45 & 159 & 116 & 86 & \\
\hline & & 32 & 159 & 116 & 62 & \\
\hline & & 22 & 157 & 111 & 38 & \\
\hline & B1 & 16 & 149 & 100 & 20 & \\
\hline & B2 & & 178 & 133 & 20 & \\
\hline & & 11 & 180 & 138 & 14 & \\
\hline & & & 148 & 97 & 12 & \\
\hline & & 8 & 180 & 138 & 8 & \\
\hline & & & 151 & 96 & 9 & \\
\hline \multirow[t]{8}{*}{ A1377 } & A & 64 & 138 & 153 & 33 & $\mathrm{~S}_{\mathrm{c}}$ \\
\hline & & 45 & 142 & 153 & 24 & \\
\hline & & 32 & 149 & 154 & 21 & \\
\hline & & 22 & 153 & 155 & 15 & \\
\hline & & 16 & 158 & 156 & 11 & \\
\hline & B & 11 & 162 & 155 & 9 & \\
\hline & $\mathrm{C}$ & & 133 & 152 & 5 & \\
\hline & & 8 & 162 & 151 & 6 & \\
\hline \multirow[t]{9}{*}{ A1401 } & A & 64 & 78 & 122 & 73 & B \\
\hline & A1 & 45 & 53 & 121 & 44 & \\
\hline & A2 & & 176 & 115 & 38 & \\
\hline & & 32 & 45 & 118 & 25 & \\
\hline & & & 175 & 117 & 23 & \\
\hline & & 22 & 169 & 121 & 14 & \\
\hline & & & 46 & 114 & 16 & \\
\hline & & 16 & 166 & 120 & 13 & \\
\hline & & 11 & 166 & 119 & 10 & \\
\hline
\end{tabular}

Table 3. continued.

\begin{tabular}{|c|c|c|c|c|c|c|}
\hline Cluster & Struct. & $a$-Scale & $X_{\mathrm{c}}$ & $Y_{\mathrm{c}}$ & $N_{\text {gal }}$ & Morph. \\
\hline & & 8 & 166 & 120 & 8 & \\
\hline A1412 & A & 64 & 108 & 109 & 29 & $\mathrm{U}$ \\
\hline \multirow[t]{7}{*}{ A1413 } & A & 64 & 162 & 131 & 66 & $\mathrm{~S}_{\mathrm{c}}$ \\
\hline & & 45 & 167 & 137 & 37 & \\
\hline & B1 & 32 & 169 & 149 & 25 & \\
\hline & B2 & & 154 & 84 & 23 & \\
\hline & & 22 & 173 & 143 & 14 & \\
\hline & & 16 & 173 & 131 & 10 & \\
\hline & & 11 & 170 & 131 & 5 & \\
\hline A1437 & A & 64 & 116 & 141 & 109 & U \\
\hline A1474 & A & 64 & 102 & 150 & 30 & $\mathrm{U}$ \\
\hline A1524 & A & 64 & 126 & 110 & 64 & $\mathrm{U}$ \\
\hline A1541 & A & 64 & 119 & 126 & 28 & $\mathrm{U}$ \\
\hline A1570 & $\mathrm{A}$ & 64 & 124 & 188 & 16 & $\mathrm{U}$ \\
\hline A1589 & A & 64 & 139 & 145 & 43 & $\mathrm{U}$ \\
\hline A1630 & A & 64 & 113 & 140 & 41 & $\mathrm{U}$ \\
\hline \multirow[t]{10}{*}{ A1631 } & A & 64 & 146 & 107 & 48 & $\mathrm{~S}_{\mathrm{c}}$ \\
\hline & & 45 & 140 & 100 & 31 & \\
\hline & & 32 & 133 & 89 & 18 & \\
\hline & B & 16 & 128 & 127 & 7 & \\
\hline & $\mathrm{C}$ & & 163 & 82 & 5 & \\
\hline & & & 169 & 177 & 6 & \\
\hline & & & 107 & 81 & 7 & \\
\hline & & 11 & 127 & 128 & 6 & \\
\hline & & & 161 & 83 & 5 & \\
\hline & & 8 & 127 & 128 & 5 & \\
\hline A1644 & A & 64 & 135 & 113 & 51 & $\mathrm{U}$ \\
\hline \multirow[t]{9}{*}{ A1650 } & A & 64 & 121 & 135 & 119 & $S_{\mathrm{f}}$ \\
\hline & & 45 & 118 & 129 & 74 & \\
\hline & & 32 & 118 & 127 & 57 & \\
\hline & & 22 & 120 & 123 & 38 & \\
\hline & & 16 & 123 & 124 & 28 & \\
\hline & B & & 178 & 89 & 10 & \\
\hline & $\mathrm{C}$ & & 97 & 35 & 10 & \\
\hline & & 11 & 125 & 126 & 16 & \\
\hline & & 8 & 125 & 127 & 12 & \\
\hline \multirow[t]{10}{*}{ A1651 } & A & 64 & 134 & 118 & 168 & $S_{\mathrm{f}}$ \\
\hline & & 45 & 135 & 122 & 109 & \\
\hline & & 32 & 136 & 124 & 68 & \\
\hline & B & & 215 & 32 & 38 & \\
\hline & & 22 & 129 & 128 & 39 & \\
\hline & & & 205 & 27 & 25 & \\
\hline & & 16 & 126 & 131 & 29 & \\
\hline & & & 203 & 26 & 20 & \\
\hline & & 11 & 128 & 130 & 19 & \\
\hline & & 8 & 129 & 129 & 13 & \\
\hline \multirow[t]{4}{*}{ A1661 } & A & 64 & 96 & 208 & 56 & $\mathrm{~S}_{\mathrm{c}}$ \\
\hline & & 45 & 77 & 217 & 31 & \\
\hline & B & 32 & 56 & 222 & 18 & \\
\hline & & 22 & 51 & 223 & 12 & \\
\hline A1675 & A & 64 & 113 & 156 & 49 & $\mathrm{U}$ \\
\hline A1689 & A & 64 & 156 & 162 & 99 & $\mathrm{U}$ \\
\hline A1691 & A & 64 & 144 & 143 & 31 & $\mathrm{U}$ \\
\hline A1700 & A & 64 & 74 & 117 & 37 & $\mathrm{U}$ \\
\hline \multirow[t]{5}{*}{ A1736 } & A & 64 & 109 & 99 & 117 & $\mathrm{~S}_{\mathrm{c}}$ \\
\hline & & 45 & 107 & 96 & 89 & \\
\hline & & 32 & 103 & 93 & 56 & \\
\hline & & 22 & 94 & 90 & 33 & \\
\hline & & & 117 & 133 & 24 & \\
\hline
\end{tabular}


P. Flin and J. Krywult: Substructures in Abell clusters of galaxies, Online Material $p 8$

Table 3. continued.

\begin{tabular}{|c|c|c|c|c|c|c|}
\hline Cluster & SStruct. & $\bar{a} a$-Scale & $\bar{~} \overline{X_{\mathrm{c}}}$ & $\overline{Y_{\mathrm{c}}}$ & $N_{\mathrm{gal}}$ & Morph. \\
\hline & $\mathrm{B}$ & 16 & 87 & 92 & 20 & \\
\hline & $\mathrm{C}$ & & 136 & 70 & 13 & \\
\hline & D & & 121 & 134 & 14 & \\
\hline & & 11 & 82 & 92 & 11 & \\
\hline & & 8 & 127 & 129 & 9 & \\
\hline & & & 114 & 149 & 8 & \\
\hline & & & 79 & 90 & 9 & \\
\hline A1749 & A & 64 & 127 & 109 & 47 & $\mathrm{U}$ \\
\hline A1774 & A & 64 & 146 & 116 & 43 & $\mathrm{U}$ \\
\hline A1775 & A & 64 & 109 & 121 & 34 & $\mathrm{U}$ \\
\hline A1795 & A & 64 & 118 & 154 & 35 & $\mathrm{U}$ \\
\hline \multirow[t]{11}{*}{ A1809 } & A & 64 & 150 & 134 & 52 & $S_{c}$ \\
\hline & & 45 & 148 & 136 & 36 & \\
\hline & & 32 & 146 & 136 & 25 & \\
\hline & B1 & 22 & 137 & 140 & 13 & \\
\hline & B2 & & 169 & 118 & 13 & \\
\hline & & 16 & 131 & 139 & 8 & \\
\hline & & 11 & 133 & 137 & 6 & \\
\hline & & & 168 & 110 & 6 & \\
\hline & & & 152 & 160 & 5 & \\
\hline & & 8 & 135 & 135 & 5 & \\
\hline & & & 168 & 109 & 5 & \\
\hline \multirow[t]{3}{*}{ A1831 } & A & 64 & 134 & 125 & 34 & $S_{\mathrm{c}}$ \\
\hline & B & 16 & 105 & 60 & 7 & \\
\hline & $\mathrm{C}$ & & 131 & 107 & 7 & \\
\hline \multirow[t]{15}{*}{ A1837 } & A & 64 & 121 & 184 & 456 & $S_{f}$ \\
\hline & & 45 & 125 & 190 & 253 & \\
\hline & & 32 & 132 & 191 & 155 & \\
\hline & B & 22 & 135 & 189 & 90 & \\
\hline & $\mathrm{C}$ & & 228 & 68 & 53 & \\
\hline & D & 16 & 134 & 189 & 55 & \\
\hline & E & & 228 & 70 & 40 & \\
\hline & & & 244 & 142 & 30 & \\
\hline & & & 129 & 130 & 44 & \\
\hline & & 11 & 246 & 142 & 21 & \\
\hline & & & 229 & 70 & 23 & \\
\hline & & & 129 & 129 & 28 & \\
\hline & & 8 & 247 & 141 & 18 & \\
\hline & & & 230 & 74 & 16 & \\
\hline & & & 128 & 128 & 18 & \\
\hline \multirow[t]{2}{*}{ A1904 } & A & 64 & 139 & 89 & 27 & $S_{\mathrm{f}}$ \\
\hline & B & 11 & 190 & 30 & 5 & \\
\hline \multirow[t]{7}{*}{ A1913 } & A & 64 & 143 & 139 & 43 & $S_{\mathrm{f}}$ \\
\hline & & 45 & 145 & 140 & 28 & \\
\hline & & 32 & 150 & 145 & 20 & \\
\hline & & 22 & 155 & 157 & 11 & \\
\hline & B & 16 & 155 & 162 & 9 & \\
\hline & $\mathrm{C}$ & & 96 & 97 & 7 & \\
\hline & & 11 & 98 & 98 & 5 & \\
\hline A1927 & A & 64 & 150 & 91 & 32 & $\mathrm{U}$ \\
\hline A1942 & A & 64 & 177 & 147 & 42 & $\mathrm{U}$ \\
\hline \multirow[t]{8}{*}{ A1983 } & A & 64 & 136 & 166 & 52 & $\mathrm{~B}, \mathrm{~S}_{\mathrm{f}}$ \\
\hline & A1 & 45 & 131 & 137 & 31 & \\
\hline & $\mathrm{A} 2$ & & 140 & 193 & 24 & \\
\hline & & 32 & 128 & 126 & 21 & \\
\hline & & 22 & 126 & 125 & 15 & \\
\hline & & & 105 & 212 & 10 & \\
\hline & & 16 & 125 & 125 & 13 & \\
\hline & B & & 108 & 207 & 7 & \\
\hline
\end{tabular}

Table 3. continued.

\begin{tabular}{|c|c|c|c|c|c|c|}
\hline Cluster & Struct. & $\overline{a-S c a l e}$ & $\overline{X_{\mathrm{c}}}$ & $\overline{Y_{\mathrm{c}}}$ & $\overline{N_{\mathrm{gal}}}$ & Morph. \\
\hline & & 11 & 126 & 125 & 8 & \\
\hline & & & 111 & 207 & 5 & \\
\hline & & 8 & 127 & 128 & 5 & \\
\hline A1991 & A & 64 & 125 & 114 & 42 & $\mathrm{U}$ \\
\hline \multirow[t]{5}{*}{ A 2020} & A & 64 & 135 & 124 & 52 & B \\
\hline & A1 & 45 & 134 & 59 & 32 & \\
\hline & A2 & & 152 & 169 & 26 & \\
\hline & & 32 & 139 & 44 & 18 & \\
\hline & & 11 & 123 & 41 & 6 & \\
\hline \multirow[t]{2}{*}{ A2022 } & A & 64 & 123 & 141 & 37 & $S_{\mathrm{f}}$ \\
\hline & B & 8 & 65 & 94 & 4 & \\
\hline \multirow[t]{8}{*}{ A2028 } & A & 64 & 143 & 174 & 23 & $S_{\mathrm{f}}$ \\
\hline & & 45 & 137 & 163 & 15 & \\
\hline & & 32 & 135 & 163 & 13 & \\
\hline & & & 33 & 230 & 7 & \\
\hline & & & 170 & 224 & 7 & \\
\hline & & 22 & 140 & 168 & 10 & \\
\hline & & & 170 & 229 & 6 & \\
\hline & B & 16 & 170 & 227 & 5 & \\
\hline A2029 & A & 64 & 125 & 135 & 73 & U \\
\hline A2040 & A & 64 & 133 & 124 & 40 & $\mathrm{U}$ \\
\hline A2048 & A & 64 & 132 & 117 & 91 & U \\
\hline \multirow[t]{9}{*}{ A2052 } & A & 64 & 117 & 157 & 93 & $S_{\mathrm{f}}$ \\
\hline & & 45 & 122 & 156 & 66 & \\
\hline & & 32 & 125 & 153 & 44 & \\
\hline & B & & 27 & 19 & 18 & \\
\hline & & 22 & 126 & 153 & 24 & \\
\hline & & & 26 & 18 & 16 & \\
\hline & & 16 & 21 & 20 & 10 & \\
\hline & & 11 & 16 & 22 & 9 & \\
\hline & & 8 & 17 & 22 & 7 & \\
\hline A2055 & A & 64 & 126 & 143 & 47 & $\mathrm{U}$ \\
\hline A2056 & A & 64 & 183 & 158 & 44 & $\mathrm{U}$ \\
\hline A2063 & A & 64 & 145 & 144 & 28 & $\mathrm{U}$ \\
\hline \multirow[t]{9}{*}{ A2065 } & A & 64 & 133 & 111 & 122 & $S_{\mathrm{f}}$ \\
\hline & & 45 & 132 & 112 & 91 & \\
\hline & & 32 & 130 & 113 & 52 & \\
\hline & & 22 & 125 & 113 & 35 & \\
\hline & B & & 238 & 233 & 12 & \\
\hline & & 16 & 121 & 114 & 18 & \\
\hline & & & 240 & 232 & 9 & \\
\hline & & 8 & 116 & 113 & 9 & \\
\hline & & & 141 & 111 & 8 & \\
\hline \multirow[t]{6}{*}{ A2073 } & A & 64 & 126 & 153 & 23 & $\mathrm{~S}_{\mathrm{f}}$ \\
\hline & & 45 & 116 & 157 & 14 & \\
\hline & & 32 & 105 & 155 & 12 & \\
\hline & & 22 & 104 & 151 & 7 & \\
\hline & B & 16 & 103 & 147 & 6 & \\
\hline & & 11 & 103 & 150 & 5 & \\
\hline A2089 & A & 64 & 115 & 108 & 26 & $\mathrm{U}$ \\
\hline A2092 & A & 64 & 120 & 135 & 27 & $\mathrm{U}$ \\
\hline A2096 & A & 64 & 95 & 140 & 33 & $\mathrm{U}$ \\
\hline A2107 & A & 64 & 139 & 125 & 51 & $\mathrm{U}$ \\
\hline \multirow[t]{6}{*}{ A2124 } & A & 64 & 112 & 71 & 46 & $\mathrm{~S}_{\mathrm{c}}$ \\
\hline & & 45 & 115 & 72 & 34 & \\
\hline & & 32 & 120 & 74 & 25 & \\
\hline & & 22 & 122 & 72 & 16 & \\
\hline & & 16 & 119 & 70 & 10 & \\
\hline & & & 114 & 99 & 8 & \\
\hline
\end{tabular}


P. Flin and J. Krywult: Substructures in Abell clusters of galaxies, Online Material $p 9$

Table 3. continued.

\begin{tabular}{|c|c|c|c|c|c|c|}
\hline$\overline{\text { Cluster }}$ & Struct. & e-Scale & $\overline{\overline{X_{\mathrm{c}}}}$ & $\overline{\bar{Y}}$ & 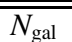 & Morph. \\
\hline & $\overline{\mathrm{B}}$ & 11 & 115 & 70 & 8 & \\
\hline & $\mathrm{C}$ & & 112 & 104 & 5 & \\
\hline & & 8 & 114 & 70 & 7 & \\
\hline A2142 & A & 64 & 100 & 123 & 30 & U \\
\hline \multirow[t]{11}{*}{ A2147 } & A & 64 & 117 & 166 & 70 & $S_{\mathrm{f}}$ \\
\hline & & 45 & 128 & 142 & 44 & \\
\hline & B1 & 32 & 135 & 132 & 28 & \\
\hline & B2 & & 139 & 223 & 22 & \\
\hline & & & 33 & 233 & 17 & \\
\hline & & 22 & 135 & 133 & 20 & \\
\hline & & & 134 & 226 & 14 & \\
\hline & & 16 & 132 & 135 & 15 & \\
\hline & & 11 & 131 & 135 & 10 & \\
\hline & & & 55 & 215 & 5 & \\
\hline & & 8 & 132 & 135 & 6 & \\
\hline \multirow[t]{8}{*}{ A2151 } & A & 64 & 114 & 141 & 69 & B \\
\hline & A1 & 45 & 128 & 128 & 44 & \\
\hline & $\mathrm{A} 2$ & & 89 & 207 & 31 & \\
\hline & & 32 & 132 & 127 & 32 & \\
\hline & & 22 & 127 & 131 & 21 & \\
\hline & & 16 & 124 & 132 & 11 & \\
\hline & & 11 & 61 & 126 & 7 & \\
\hline & & 8 & 60 & 127 & 5 & \\
\hline \multirow[t]{13}{*}{ A2152 } & A & 64 & 148 & 118 & 68 & $\mathrm{~B}, \mathrm{~S}_{\mathrm{f}}$ \\
\hline & A1 & 45 & 111 & 106 & 34 & \\
\hline & A2 & & 151 & 143 & 34 & \\
\hline & & 32 & 102 & 98 & 22 & \\
\hline & & 22 & 163 & 160 & 15 & \\
\hline & B & & 207 & 105 & 11 & \\
\hline & & & 103 & 93 & 13 & \\
\hline & & 16 & 206 & 104 & 10 & \\
\hline & & & 171 & 173 & 10 & \\
\hline & & 11 & 208 & 102 & 6 & \\
\hline & & & 118 & 121 & 9 & \\
\hline & & & 172 & 174 & 7 & \\
\hline & & & 149 & 151 & 4 & \\
\hline A2175 & A & 64 & 99 & 109 & 66 & $\mathrm{U}$ \\
\hline A2197 & A & 64 & 91 & 99 & 67 & $\mathrm{U}$ \\
\hline \multirow[t]{14}{*}{ A2199 } & A & 64 & 137 & 126 & 97 & $S_{\mathrm{f}}$ \\
\hline & & 45 & 129 & 130 & 59 & \\
\hline & & 32 & 125 & 135 & 42 & \\
\hline & & 22 & 125 & 133 & 27 & \\
\hline & & 16 & 126 & 129 & 20 & \\
\hline & & 11 & 127 & 128 & 13 & \\
\hline & B & & 152 & 85 & 8 & \\
\hline & & & 161 & 246 & 5 & \\
\hline & & & 209 & 151 & 5 & \\
\hline & & & 69 & 181 & 7 & \\
\hline & & 8 & 126 & 127 & 8 & \\
\hline & $\mathrm{C}$ & & 70 & 181 & 6 & \\
\hline & & & 150 & 82 & 6 & \\
\hline & & & 211 & 153 & 5 & \\
\hline A2210 & A & 64 & 162 & 155 & 64 & $\mathrm{U}$ \\
\hline \multirow[t]{2}{*}{ A2255 } & A & 64 & 113 & 113 & 42 & $S_{\mathrm{c}}$ \\
\hline & B & 16 & 98 & 113 & 9 & \\
\hline A2256 & A & 64 & 119 & 107 & 61 & $\mathrm{U}$ \\
\hline A2311 & A & 64 & 114 & 119 & 32 & $\mathrm{U}$ \\
\hline \multirow[t]{2}{*}{ A2312 } & A & 64 & 120 & 136 & 26 & $S_{\mathrm{c}}$ \\
\hline & B & 11 & 131 & 167 & 4 & \\
\hline
\end{tabular}

Table 3. continued.

\begin{tabular}{|c|c|c|c|c|c|c|}
\hline Cluster & Struct. & $a$-Scale & $\overline{X_{\mathrm{c}}}$ & $Y_{\mathrm{c}}$ & $\overline{N_{\text {gal }}}$ & Morph. \\
\hline A2315 & $\overline{\mathrm{A}}$ & 64 & 80 & 102 & 15 & $\mathrm{U}$ \\
\hline A2319 & A & 64 & 175 & 155 & 25 & $\mathrm{U}$ \\
\hline A2366 & A & 64 & 125 & 126 & 30 & $\mathrm{U}$ \\
\hline A2384 & A & 64 & 126 & 142 & 25 & $\mathrm{U}$ \\
\hline A2399 & A & 64 & 136 & 117 & 34 & $\mathrm{U}$ \\
\hline A2410 & A & 64 & 145 & 131 & 29 & $\mathrm{U}$ \\
\hline \multirow[t]{2}{*}{ A2457 } & A & 64 & 130 & 132 & 54 & $S_{\mathrm{c}}$ \\
\hline & B & 11 & 141 & 82 & 6 & \\
\hline \multirow[t]{10}{*}{ A2593 } & A & 64 & 144 & 138 & 67 & $\mathrm{~S}_{\mathrm{f}}$ \\
\hline & & 45 & 141 & 140 & 48 & \\
\hline & & 32 & 139 & 142 & 39 & \\
\hline & & 22 & 137 & 145 & 31 & \\
\hline & & 16 & 135 & 145 & 23 & \\
\hline & & 11 & 130 & 143 & 13 & \\
\hline & B & & 150 & 83 & 6 & \\
\hline & & 8 & 127 & 142 & 10 & \\
\hline & & & 150 & 84 & 5 & \\
\hline & & & 144 & 152 & 6 & \\
\hline A2597 & A & 64 & 166 & 164 & 27 & $\mathrm{U}$ \\
\hline A2634 & A & 64 & 111 & 150 & 163 & $\mathrm{U}$ \\
\hline A2657 & A & 64 & 135 & 134 & 52 & $\mathrm{U}$ \\
\hline A2665 & A & 64 & 91 & 139 & 25 & $\mathrm{U}$ \\
\hline A2666 & A & 64 & 66 & 86 & 22 & $\mathrm{U}$ \\
\hline A2670 & A & 64 & 134 & 140 & 181 & $\mathrm{U}$ \\
\hline A2700 & A & 64 & 118 & 145 & 30 & $\mathrm{U}$ \\
\hline A2717 & A & 64 & 136 & 119 & 39 & $\mathrm{U}$ \\
\hline A2721 & A & 64 & 105 & 142 & 51 & $\mathrm{U}$ \\
\hline \multirow[t]{8}{*}{ A2877 } & A & 64 & 142 & 124 & 83 & $\mathrm{~S}_{\mathrm{c}}$ \\
\hline & & 45 & 141 & 125 & 60 & \\
\hline & & 32 & 140 & 125 & 37 & \\
\hline & & 22 & 140 & 124 & 25 & \\
\hline & & 16 & 140 & 123 & 21 & \\
\hline & & 11 & 140 & 124 & 17 & \\
\hline & B & & 167 & 154 & 7 & \\
\hline & & 8 & 140 & 129 & 9 & \\
\hline \multirow[t]{15}{*}{ A3128 } & A & 64 & 133 & 112 & 160 & $\mathrm{~S}_{c, f}$ \\
\hline & & 45 & 128 & 117 & 117 & \\
\hline & & 32 & 128 & 118 & 82 & \\
\hline & B & & 207 & 18 & 28 & \\
\hline & & 22 & 129 & 119 & 61 & \\
\hline & & & 208 & 17 & 15 & \\
\hline & & 16 & 129 & 119 & 38 & \\
\hline & $\mathrm{C}$ & & 210 & 93 & 12 & \\
\hline & D & 11 & 140 & 115 & 20 & \\
\hline & $\mathrm{E}$ & & 209 & 92 & 10 & \\
\hline & $\mathrm{F}$ & & 118 & 120 & 16 & \\
\hline & & 8 & 141 & 114 & 11 & \\
\hline & & & 209 & 92 & 10 & \\
\hline & & & 115 & 120 & 10 & \\
\hline & & & 118 & 141 & 10 & \\
\hline A3266 & A & 64 & 102 & 163 & 28 & $\mathrm{U}$ \\
\hline \multirow[t]{7}{*}{ A3376 } & A & 64 & 164 & 119 & 43 & $\mathrm{~S}_{\mathrm{c}}$ \\
\hline & & 45 & 170 & 114 & 27 & \\
\hline & & 32 & 182 & 110 & 20 & \\
\hline & B & 22 & 188 & 109 & 11 & \\
\hline & & & 128 & 128 & 8 & \\
\hline & & 16 & 125 & 127 & 8 & \\
\hline & $\mathrm{C}$ & 11 & 126 & 125 & 6 & \\
\hline A3391 & A & 64 & 127 & 67 & 21 & $\mathrm{U}$ \\
\hline
\end{tabular}


P. Flin and J. Krywult: Substructures in Abell clusters of galaxies, Online Material p 10

Table 3. continued.

\begin{tabular}{|c|c|c|c|c|c|c|}
\hline Cluster & Struct. & $a$-Scale & $X_{\mathrm{c}}$ & $Y_{\mathrm{c}}$ & $\begin{array}{l}N_{\mathrm{gal}} \\
\end{array}$ & Morph. \\
\hline \multirow[t]{9}{*}{ A3395 } & $\bar{A}$ & 64 & 147 & 143 & 24 & B \\
\hline & A1 & 45 & 137 & 124 & 16 & \\
\hline & A2 & & 191 & 232 & 13 & \\
\hline & & 32 & 188 & 237 & 9 & \\
\hline & & & 140 & 125 & 11 & \\
\hline & & 22 & 188 & 240 & 8 & \\
\hline & & & 139 & 130 & 8 & \\
\hline & & 16 & 186 & 241 & 8 & \\
\hline & & 11 & 185 & 240 & 6 & \\
\hline \multirow[t]{10}{*}{ A3526 } & A & 64 & 124 & 111 & 40 & B \\
\hline & A1 & 45 & 94 & 116 & 31 & \\
\hline & A2 & & 202 & 111 & 23 & \\
\hline & & 32 & 90 & 117 & 20 & \\
\hline & & & 199 & 109 & 19 & \\
\hline & & 22 & 90 & 115 & 13 & \\
\hline & & & 196 & 107 & 9 & \\
\hline & & 16 & 90 & 113 & 11 & \\
\hline & & & 211 & 112 & 7 & \\
\hline & & 11 & 215 & 114 & 5 & \\
\hline A3558 & A & 64 & 99 & 101 & 79 & $\mathrm{U}$ \\
\hline \multirow[t]{13}{*}{ A 3562} & A & 64 & 185 & 119 & 108 & $\mathrm{~S}_{c, f}$ \\
\hline & & 45 & 198 & 125 & 70 & \\
\hline & & & 34 & 73 & 44 & \\
\hline & & 32 & 204 & 132 & 49 & \\
\hline & & & 27 & 64 & 28 & \\
\hline & & 22 & 205 & 133 & 34 & \\
\hline & B & & 24 & 60 & 17 & \\
\hline & & 16 & 202 & 129 & 22 & \\
\hline & & & 22 & 62 & 14 & \\
\hline & $\mathrm{C} 1$ & 11 & 197 & 121 & 14 & \\
\hline & $\mathrm{C} 2$ & & 215 & 141 & 12 & \\
\hline & & 8 & 197 & 119 & 8 & \\
\hline & & & 214 & 141 & 9 & \\
\hline \multirow[t]{11}{*}{ A3667 } & A & 64 & 82 & 90 & 93 & $\mathrm{~S}_{c, f}$ \\
\hline & & 45 & 64 & 74 & 58 & \\
\hline & B & 32 & 59 & 76 & 37 & \\
\hline & & & 128 & 126 & 36 & \\
\hline & & 22 & 60 & 80 & 25 & \\
\hline & $\mathrm{C}$ & & 127 & 123 & 18 & \\
\hline & & 16 & 61 & 82 & 19 & \\
\hline & & 11 & 60 & 80 & 11 & \\
\hline & D1 & 8 & 57 & 76 & 6 & \\
\hline & D2 & & 66 & 90 & 7 & \\
\hline & & & 21 & 65 & 6 & \\
\hline A3716 & A & 64 & 139 & 128 & 73 & $\mathrm{U}$ \\
\hline A3888 & A & 64 & 133 & 129 & 31 & $\mathrm{U}$ \\
\hline A4038 & A & 64 & 133 & 129 & 101 & $\mathrm{U}$ \\
\hline
\end{tabular}


P. Flin and J. Krywult: Substructures in Abell clusters of galaxies, Online Material $p 11$

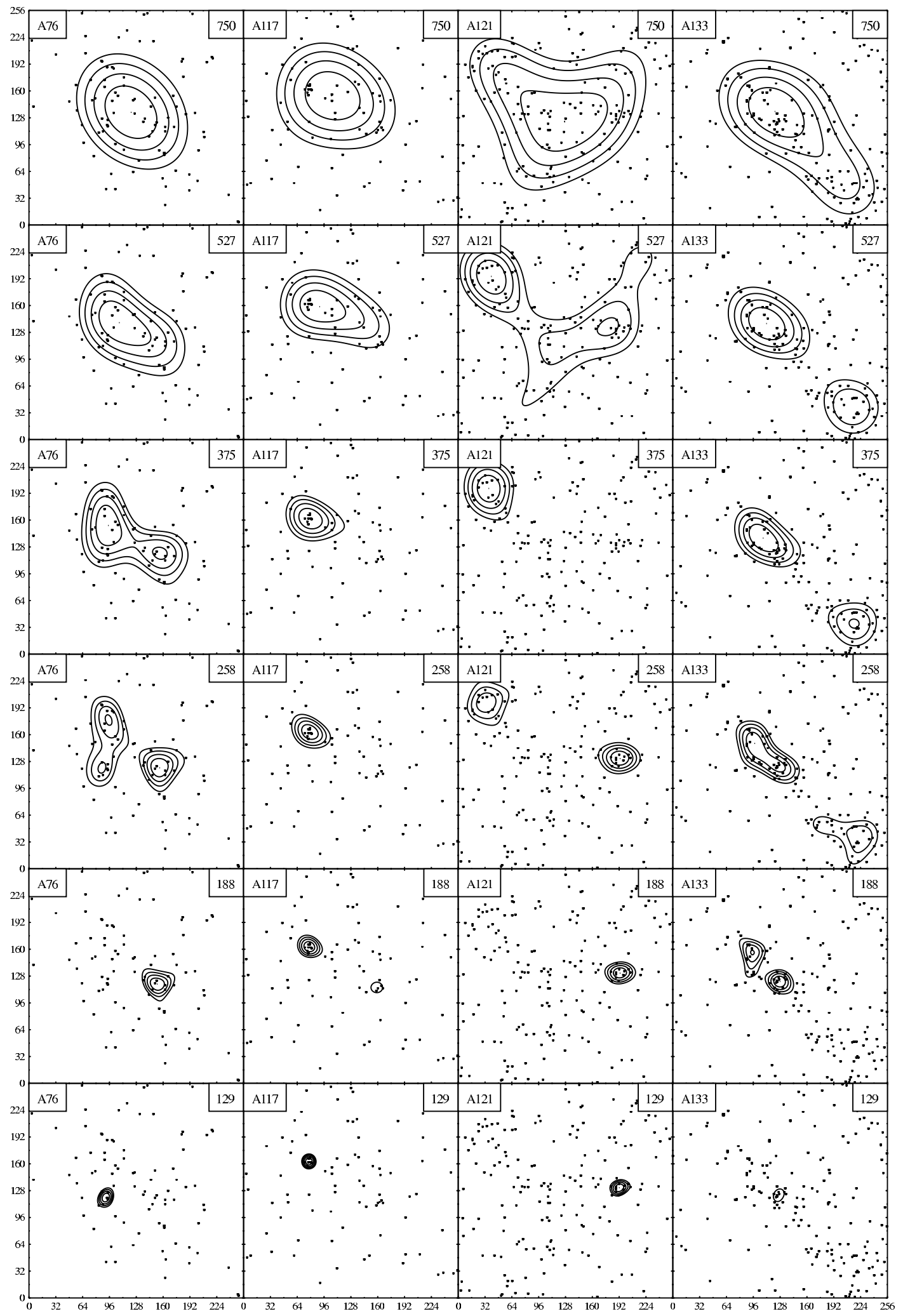

Fig. 3. Distribution of galaxies in A76, A117, A121 and A133 with wavelet images for scales $a=129,188,258,375,527,750$ kpc presented in the upper right box. 
P. Flin and J. Krywult: Substructures in Abell clusters of galaxies, Online Material $p 12$

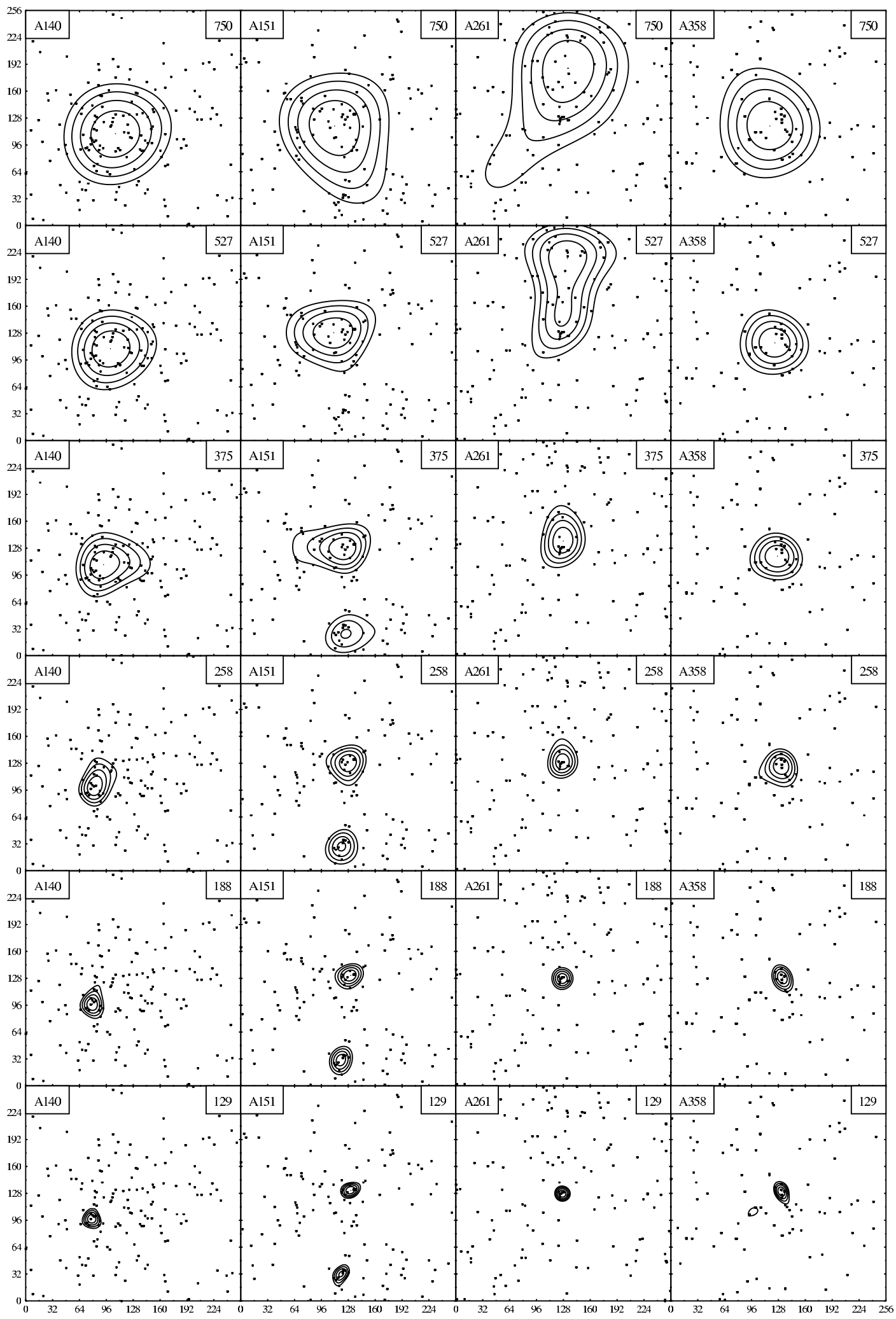

Fig. 4. Distribution of galaxies in A140, A151, A261 and A358 with wavelet images for scales $a=129,188,258,375,527,750 \mathrm{kpc}$ presented in the upper right box. 
P. Flin and J. Krywult: Substructures in Abell clusters of galaxies, Online Material p 13

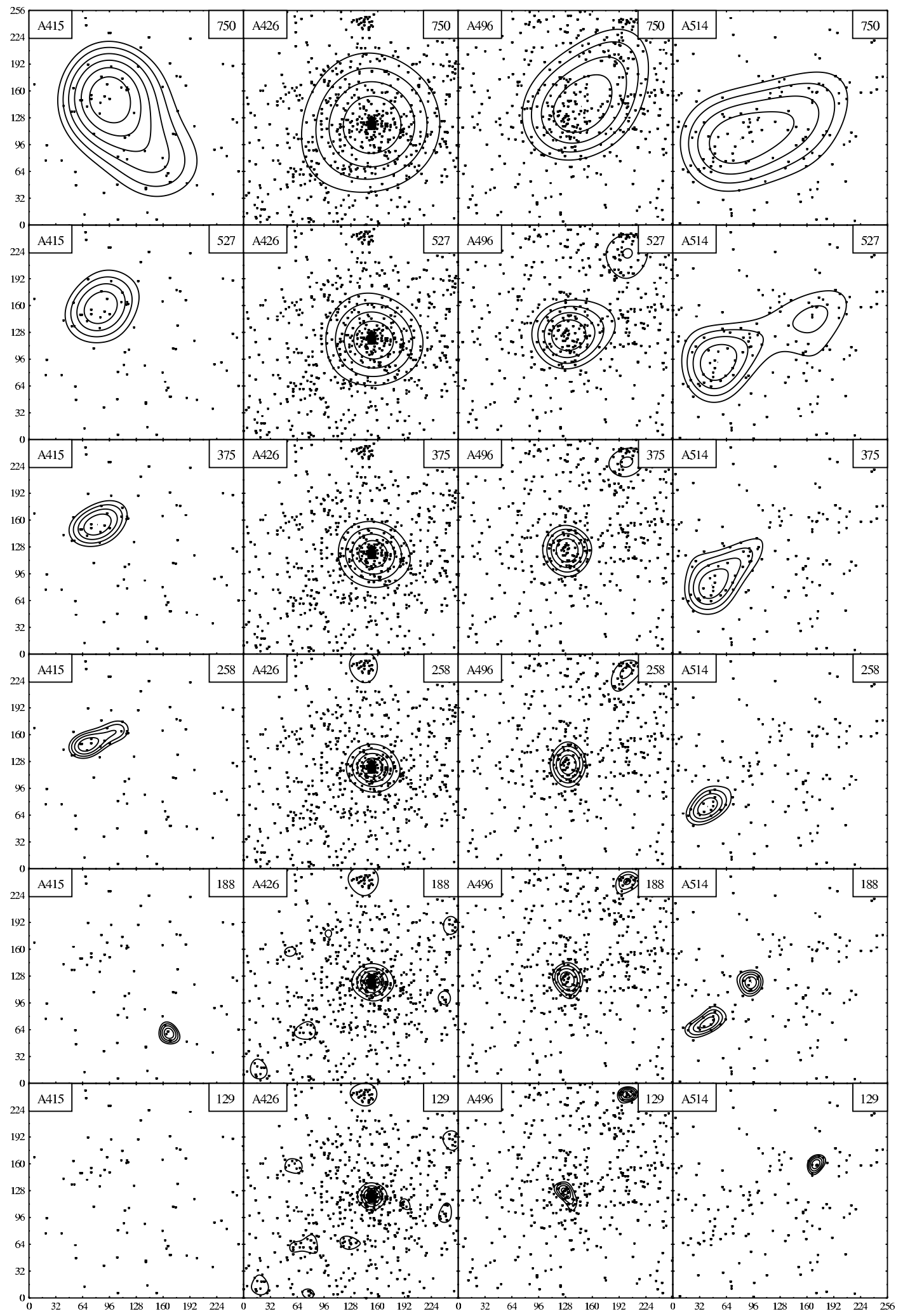

Fig. 5. Distribution of galaxies in A415, A426, A496 and A514 with wavelet images for scales $a=129,188,258,375,527,750$ kpc presented in the upper right box. 
P. Flin and J. Krywult: Substructures in Abell clusters of galaxies, Online Material p 14

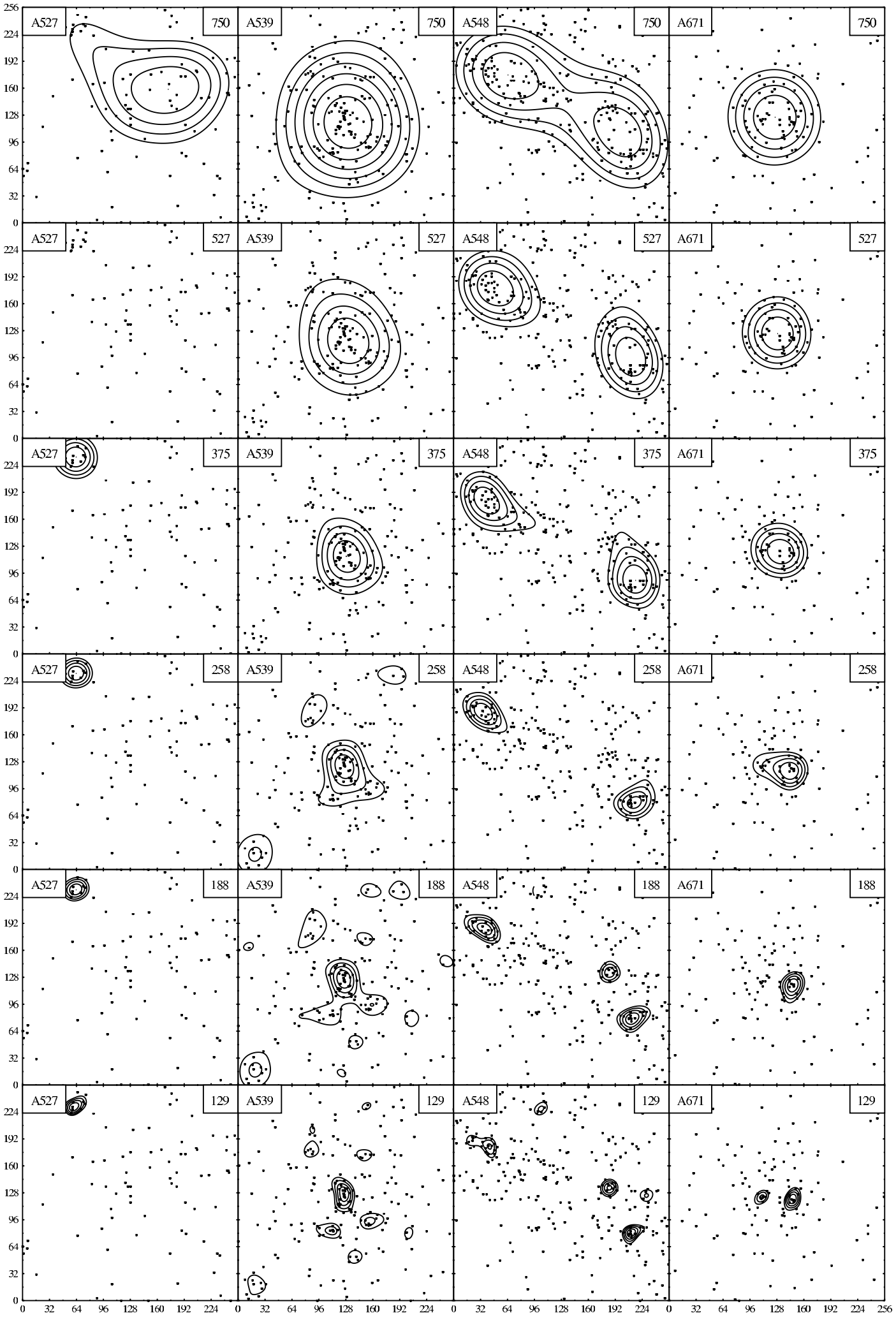

Fig. 6. Distribution of galaxies in A527, A539, A548 and A671 with wavelet images for scales $a=129,188,258,375,527,750 \mathrm{kpc}$ presented in the upper right box. 
P. Flin and J. Krywult: Substructures in Abell clusters of galaxies, Online Material p 15

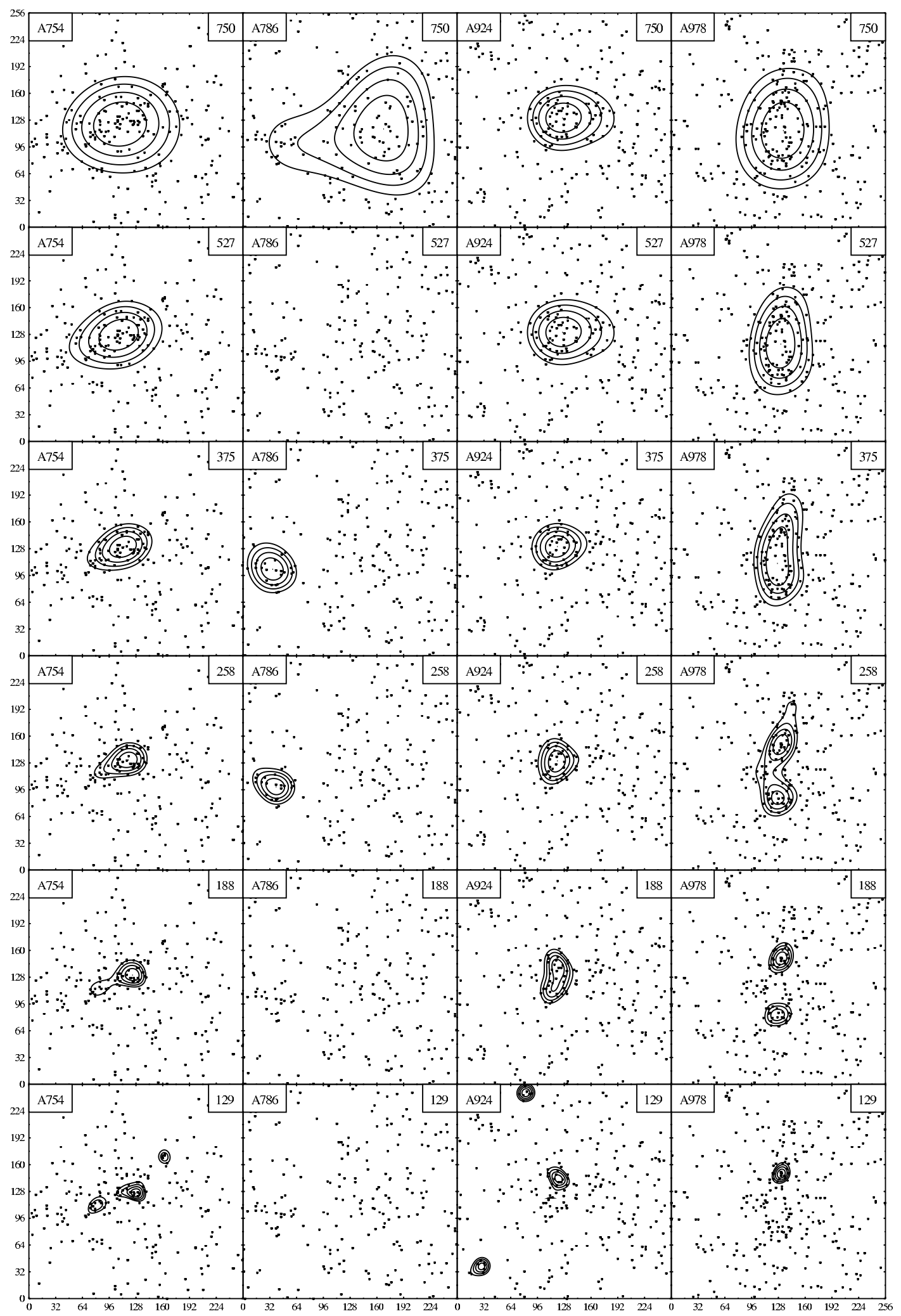

Fig. 7. Distribution of galaxies in A754, A786, A924 and A978 with wavelet images for scales $a=129,188,258,375,527,750$ kpc presented in the upper right box. 
P. Flin and J. Krywult: Substructures in Abell clusters of galaxies, Online Material p 16

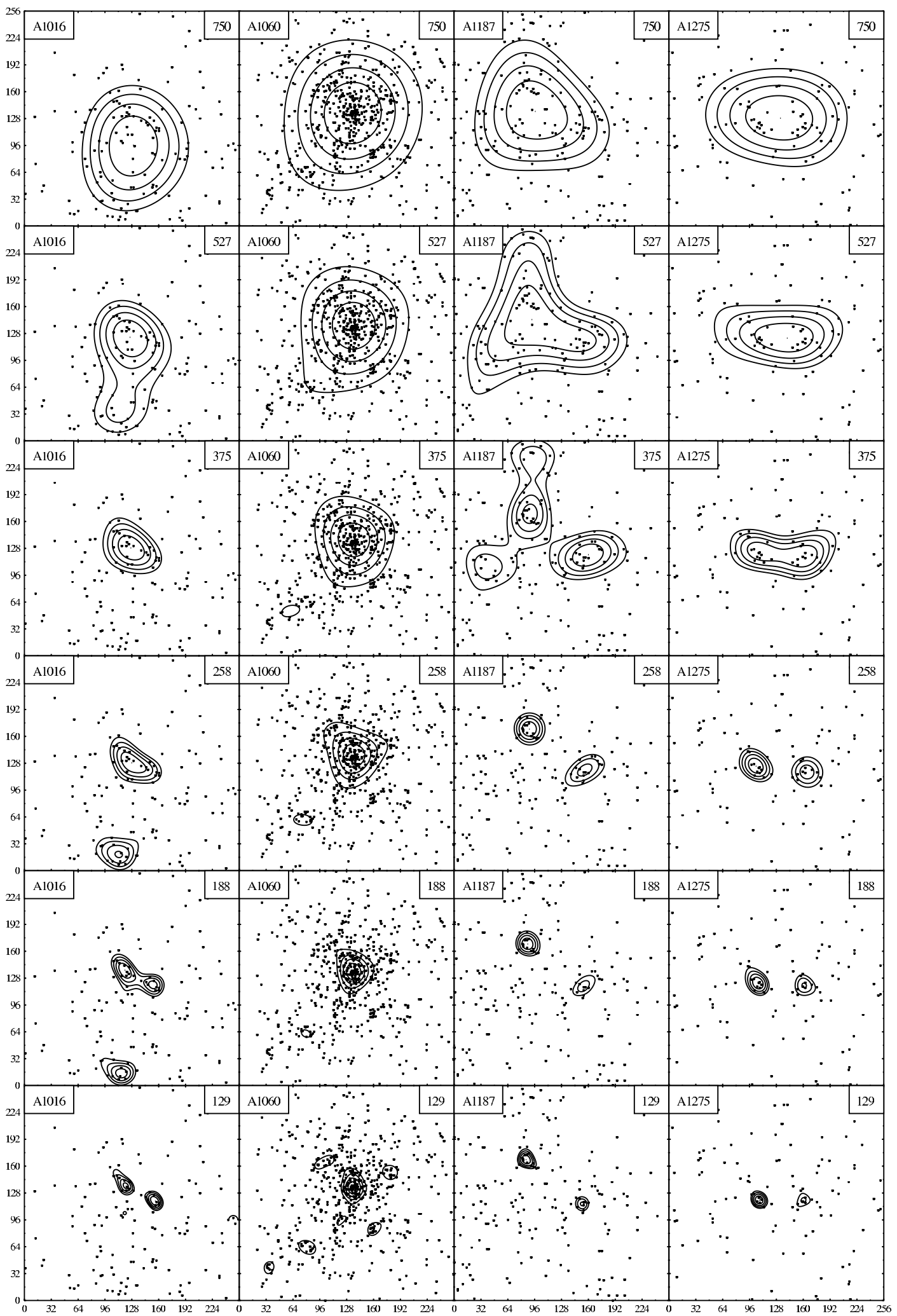

Fig. 8. Distribution of galaxies in A1016, A1060, A1187 and A1275 with wavelet images for scales $a=129,188,258,375,527,750 \mathrm{kpc}$ presented in the upper right box. 
P. Flin and J. Krywult: Substructures in Abell clusters of galaxies, Online Material p 17

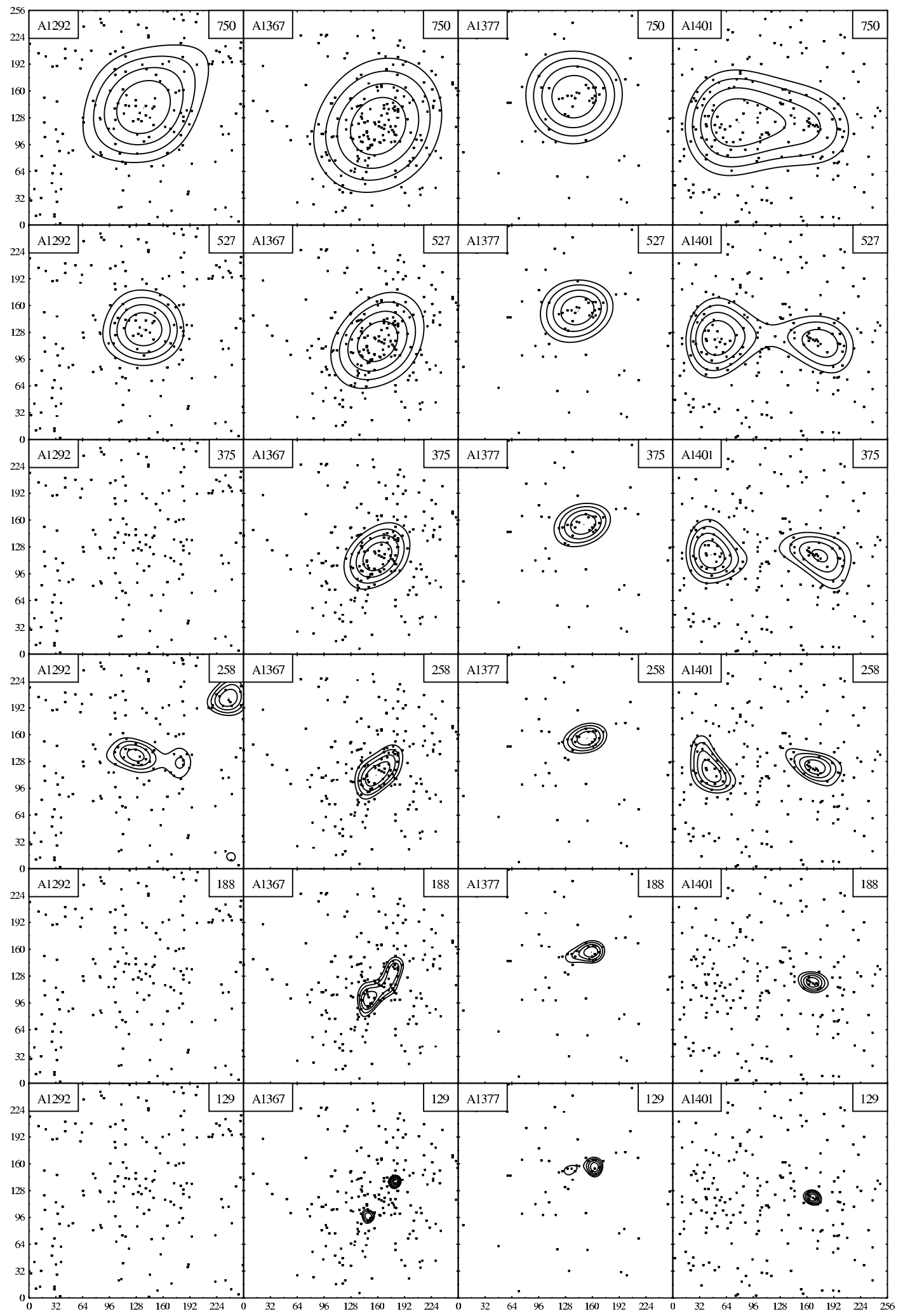

Fig. 9. Distribution of galaxies in A1292, A1367, A1377 and A1401 with wavelet images for scales $a=129,188,258,375,527,750 \mathrm{kpc}$ presented in the upper right box. 
P. Flin and J. Krywult: Substructures in Abell clusters of galaxies, Online Material $p 18$

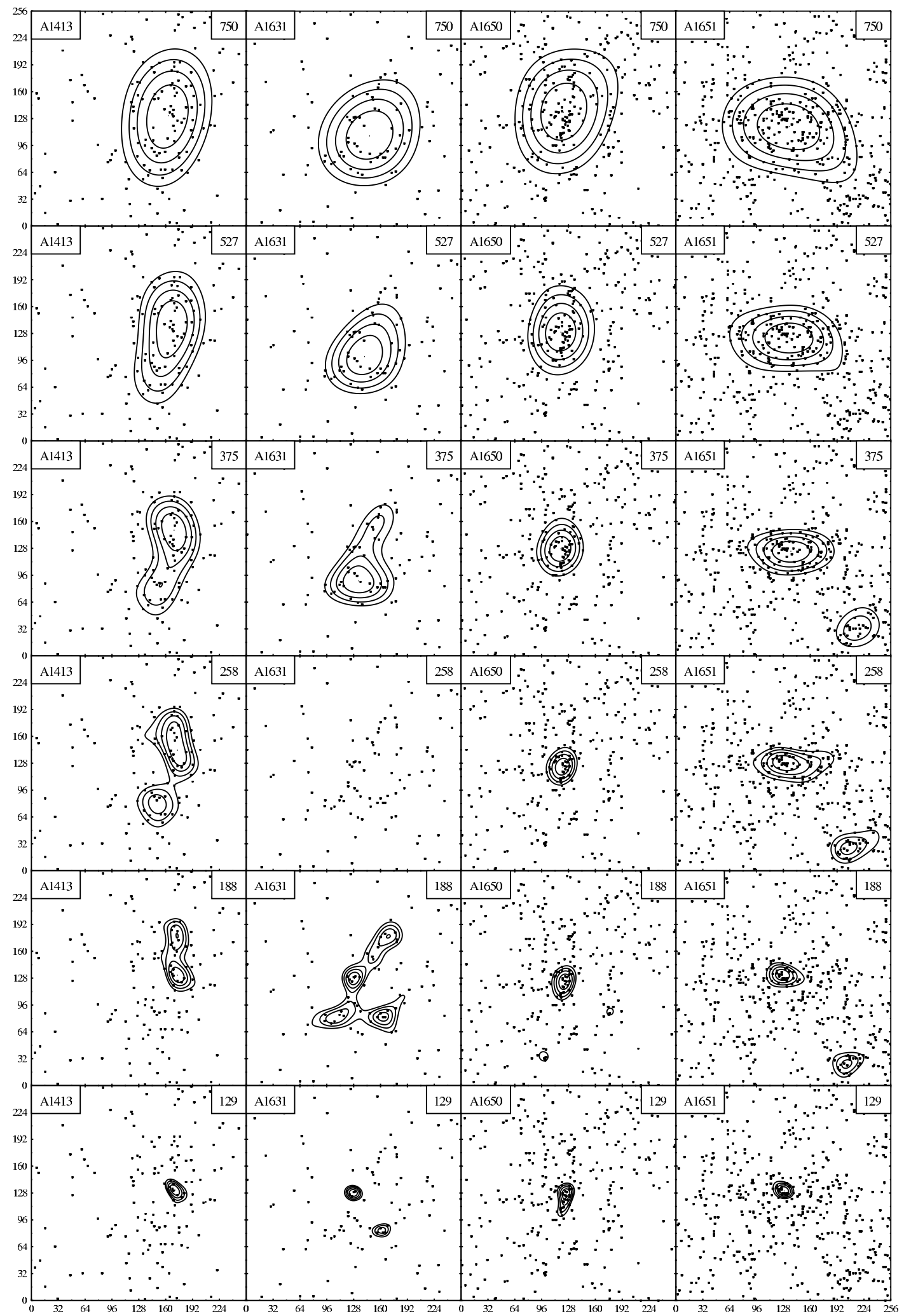

Fig. 10. Distribution of galaxies in A1413, A1631, A1650 and A1651 with wavelet images for scales $a=129,188,258,375,527,750 \mathrm{kpc}$ presented in the upper right box. 
P. Flin and J. Krywult: Substructures in Abell clusters of galaxies, Online Material $p 19$

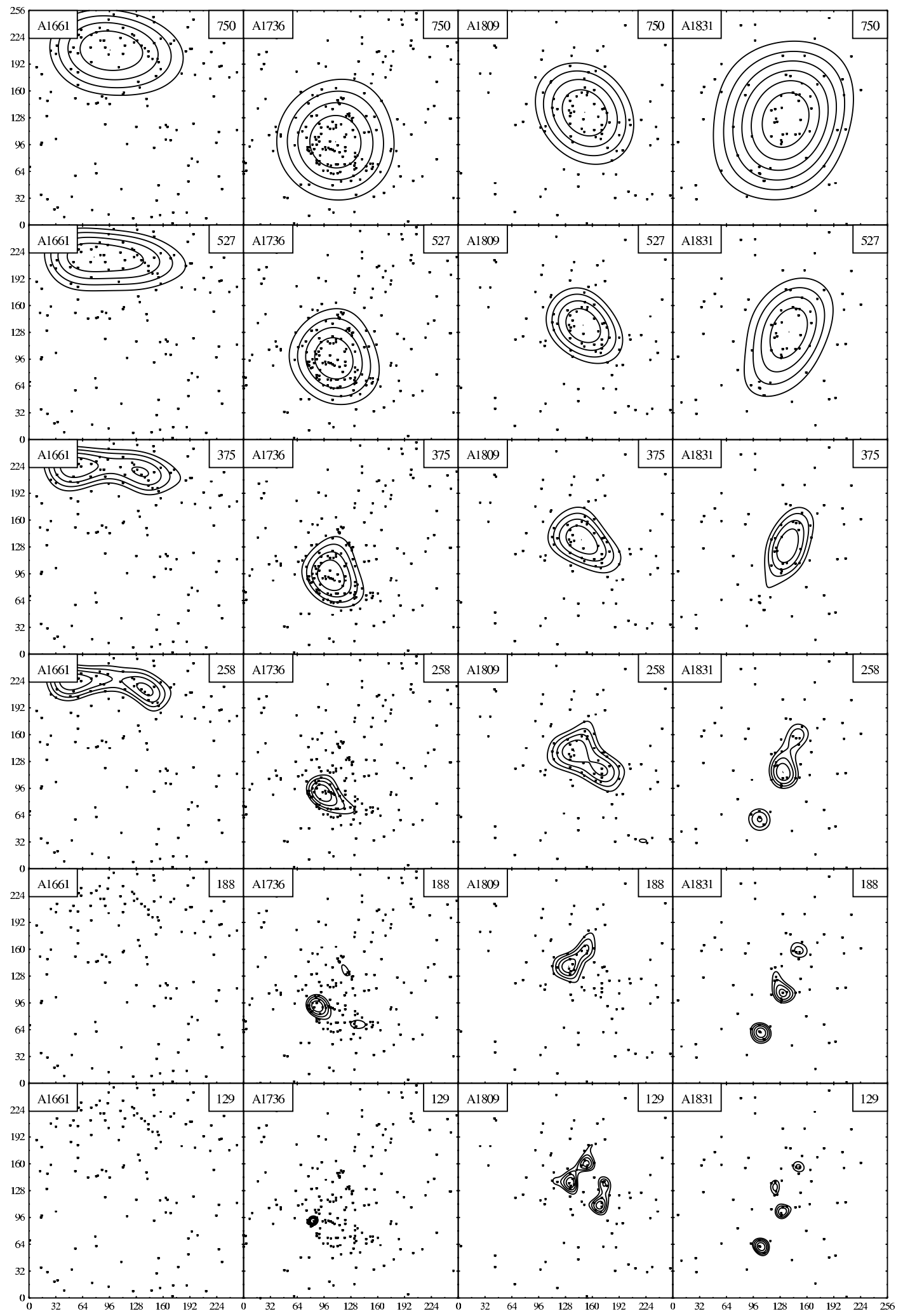

Fig. 11. Distribution of galaxies in A1661, A1736, A1809 and A1831 with wavelet images for scales $a=129,188,258,375,527,750 \mathrm{kpc}$ presented in the upper right box. 
P. Flin and J. Krywult: Substructures in Abell clusters of galaxies, Online Material p 20

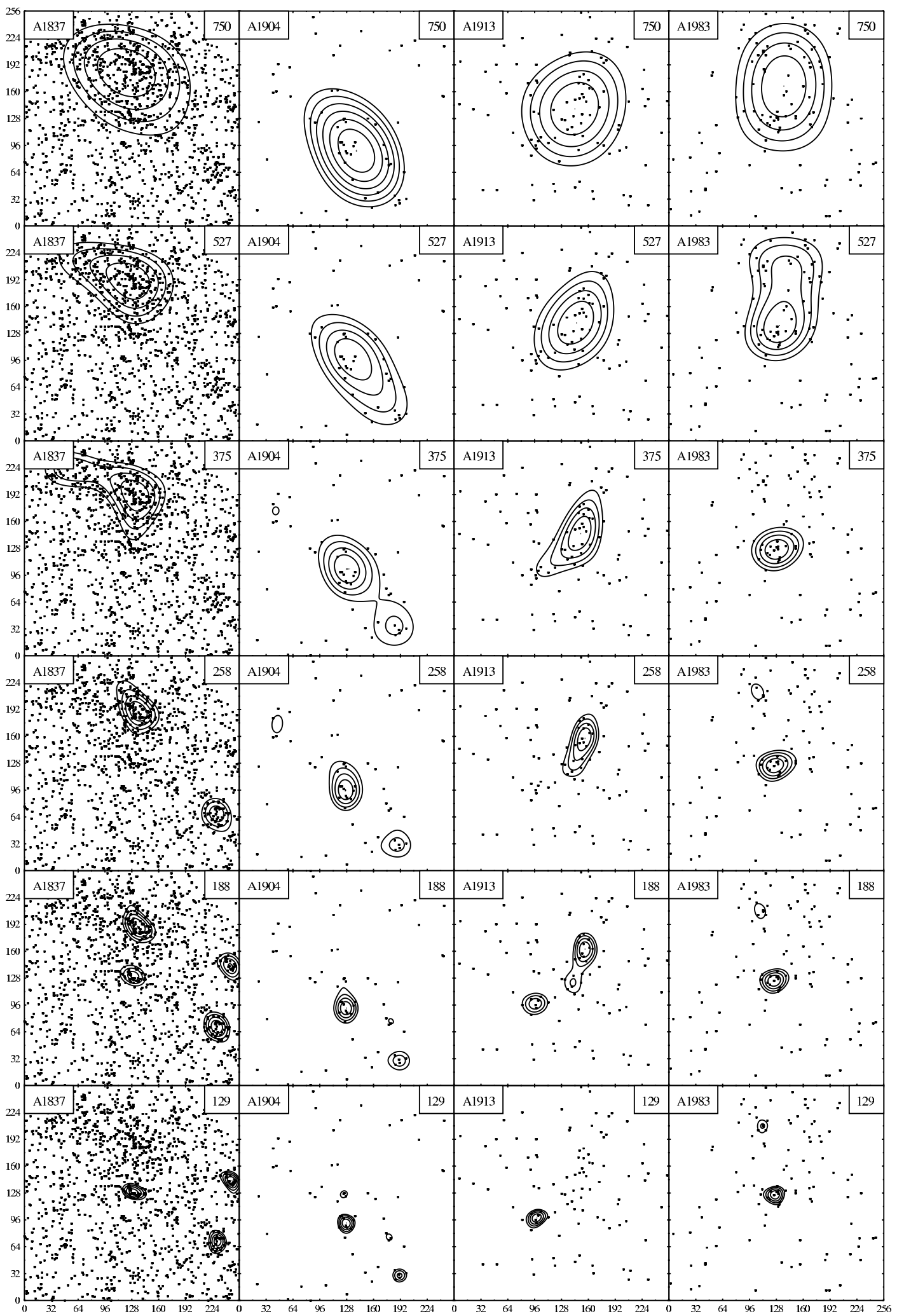

Fig. 12. Distribution of galaxies in A1837, A1904, A1913 and A1983 with wavelet images for scales $a=129,188,258,375,527,750 \mathrm{kpc}$ presented in the upper right box. 
P. Flin and J. Krywult: Substructures in Abell clusters of galaxies, Online Material p 21

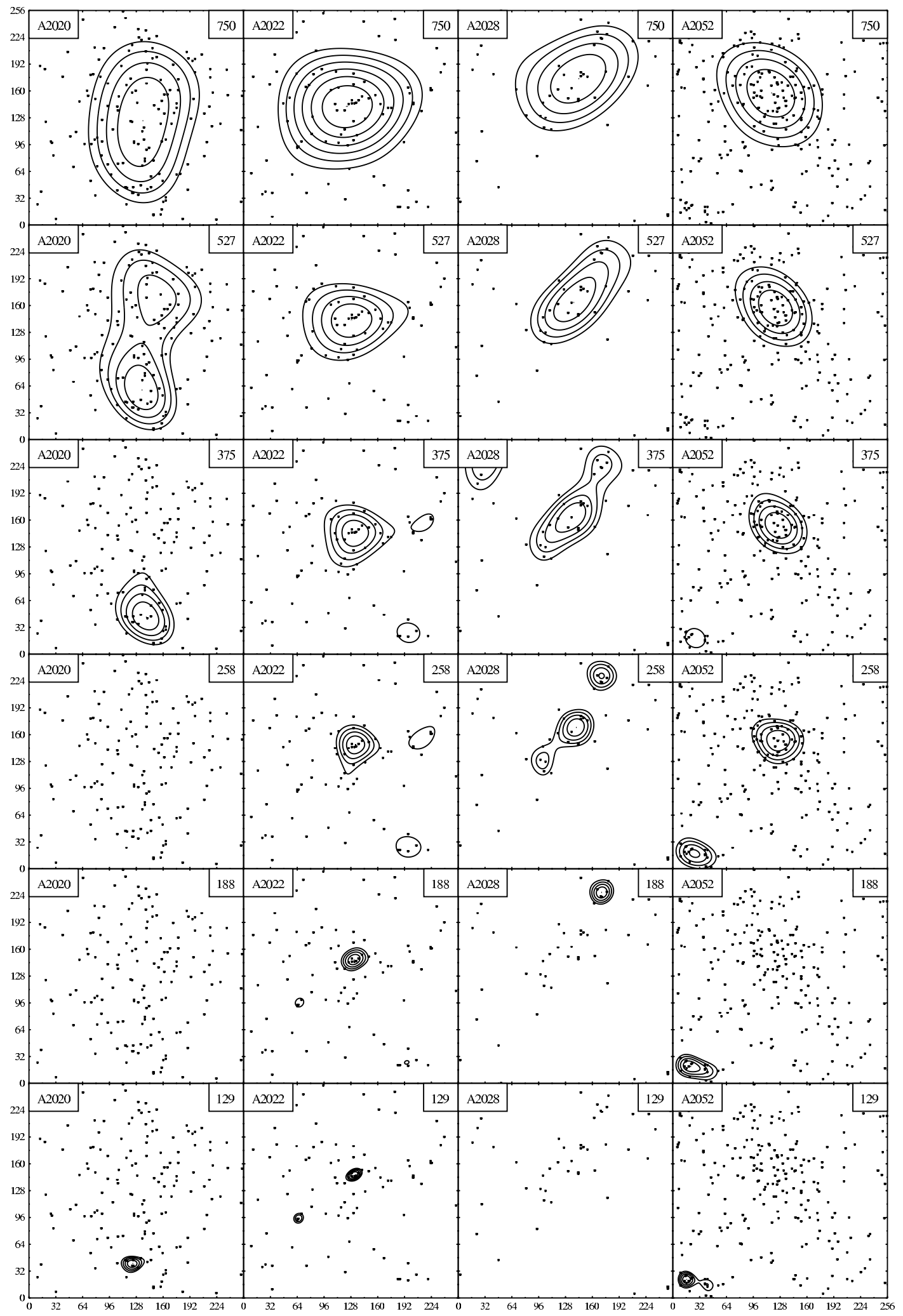

Fig. 13. Distribution of galaxies in A2020, A2022, A2028 and A2052 with wavelet images for scales $a=129,188,258,375,527,750 \mathrm{kpc}$ presented in the upper right box. 
P. Flin and J. Krywult: Substructures in Abell clusters of galaxies, Online Material p 22

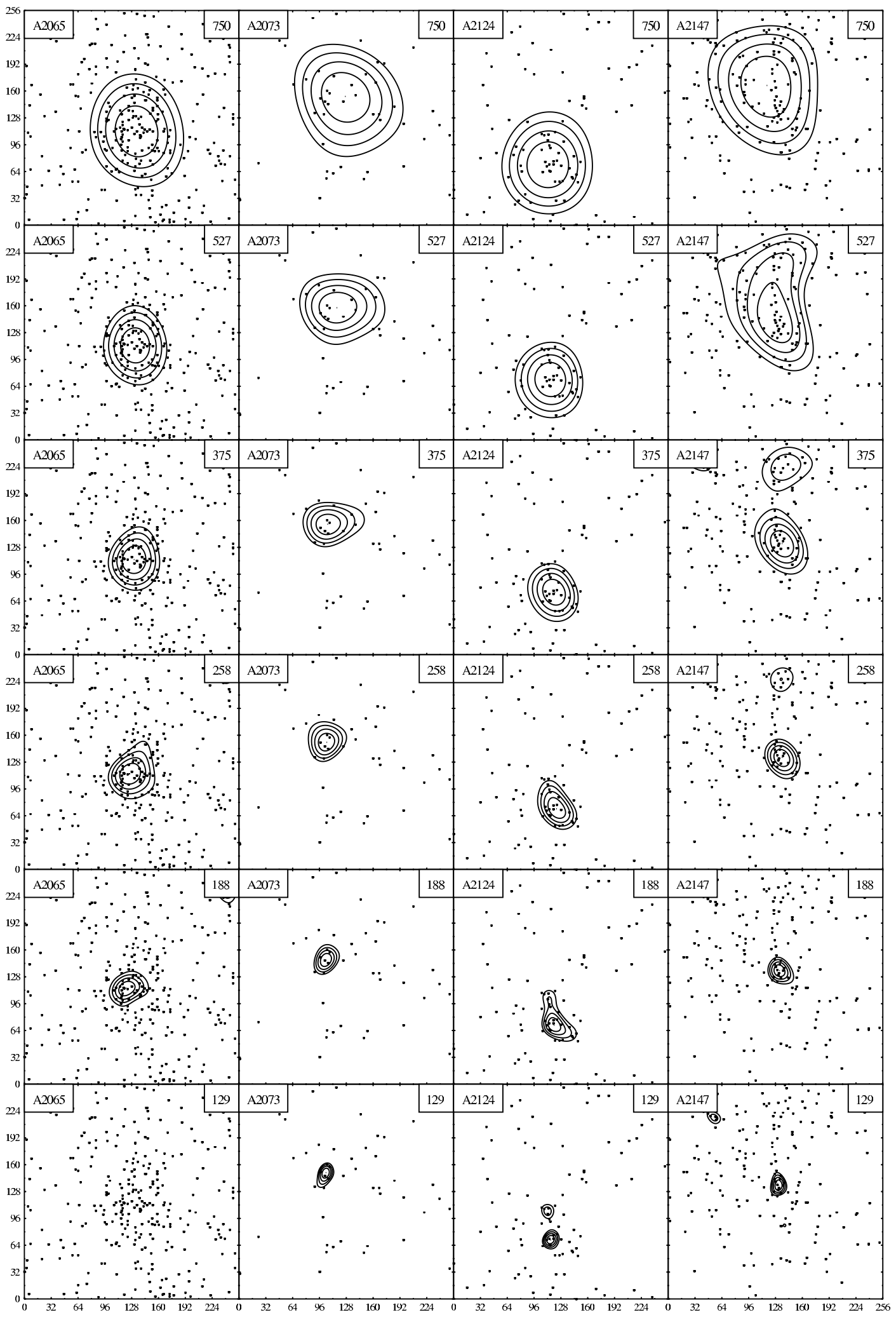

Fig. 14. Distribution of galaxies in A2065, A2073, A2124 and A2147 with wavelet images for scales $a=129,188,258,375,527,750$ kpc presented in the upper right box. 
P. Flin and J. Krywult: Substructures in Abell clusters of galaxies, Online Material p 23

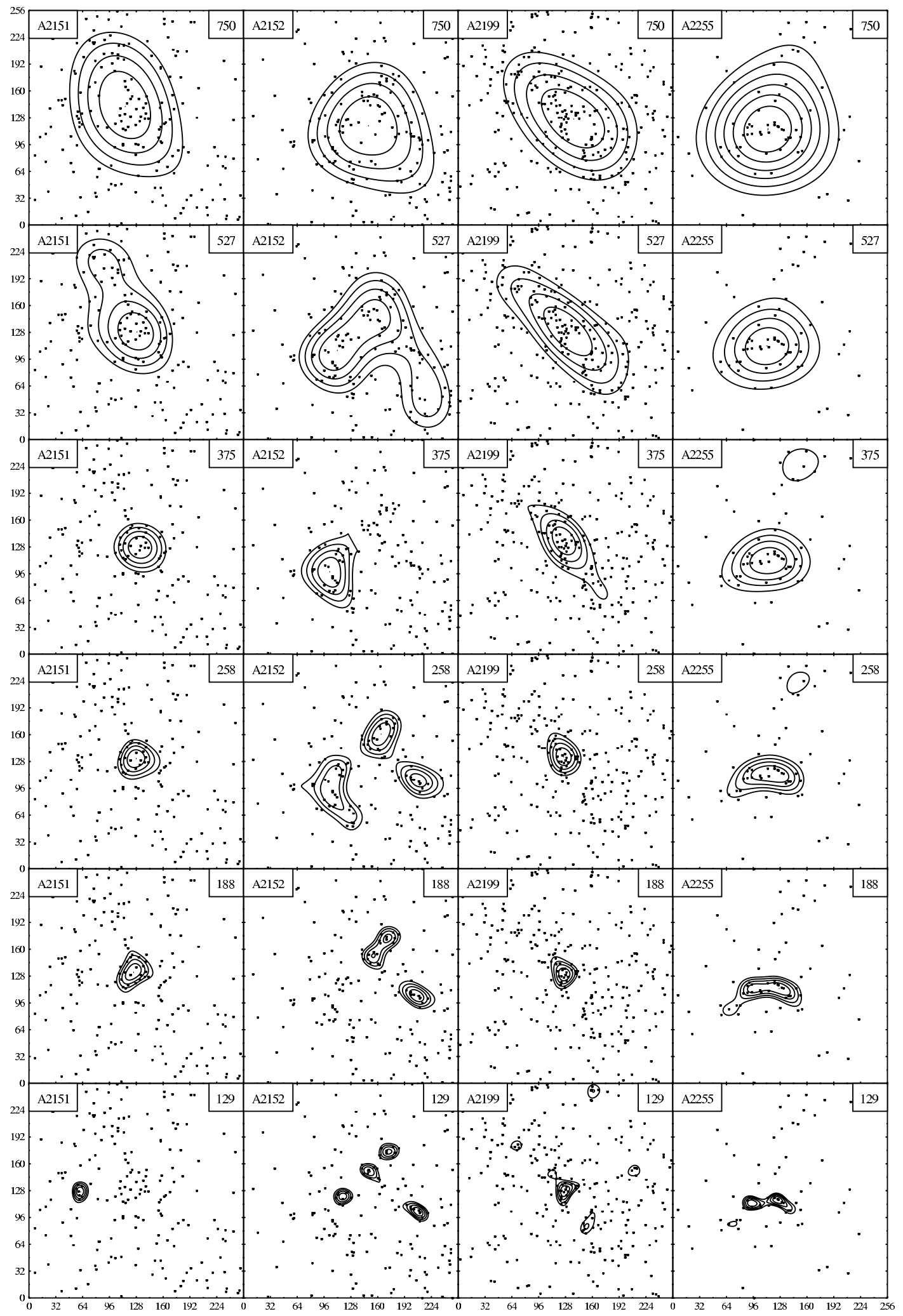

Fig. 15. Distribution of galaxies in A2151, A2152, A2199 and A2255 with wavelet images for scales $a=129,188,258,375,527,750 \mathrm{kpc}$ presented in the upper right box. 
P. Flin and J. Krywult: Substructures in Abell clusters of galaxies, Online Material $p 24$

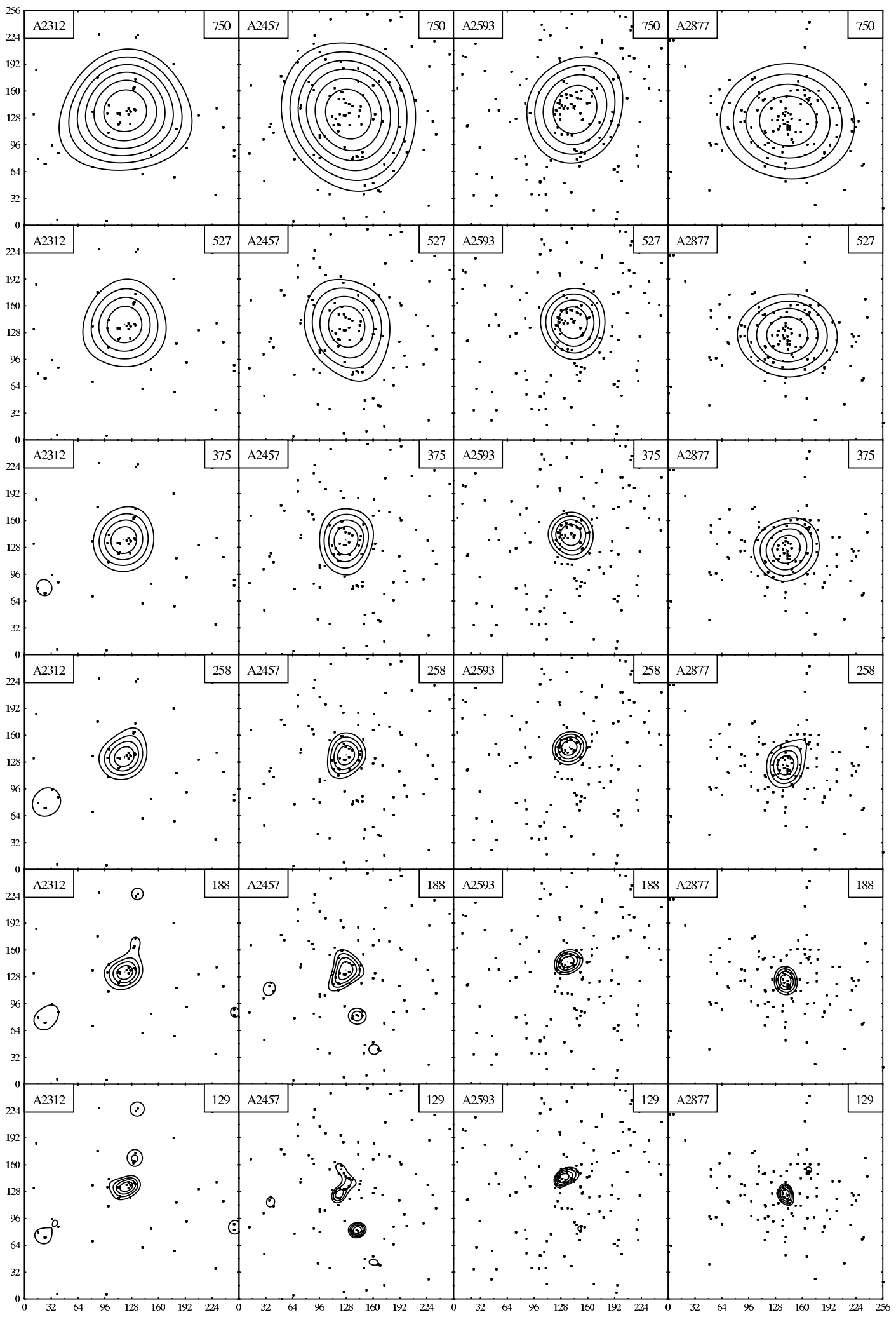

Fig. 16. Distribution of galaxies in A2312, A2457, A2593 and A2877 with wavelet images for scales $a=129,188,258,375,527,750 \mathrm{kpc}$ presented in the upper right box. 
P. Flin and J. Krywult: Substructures in Abell clusters of galaxies, Online Material p 25

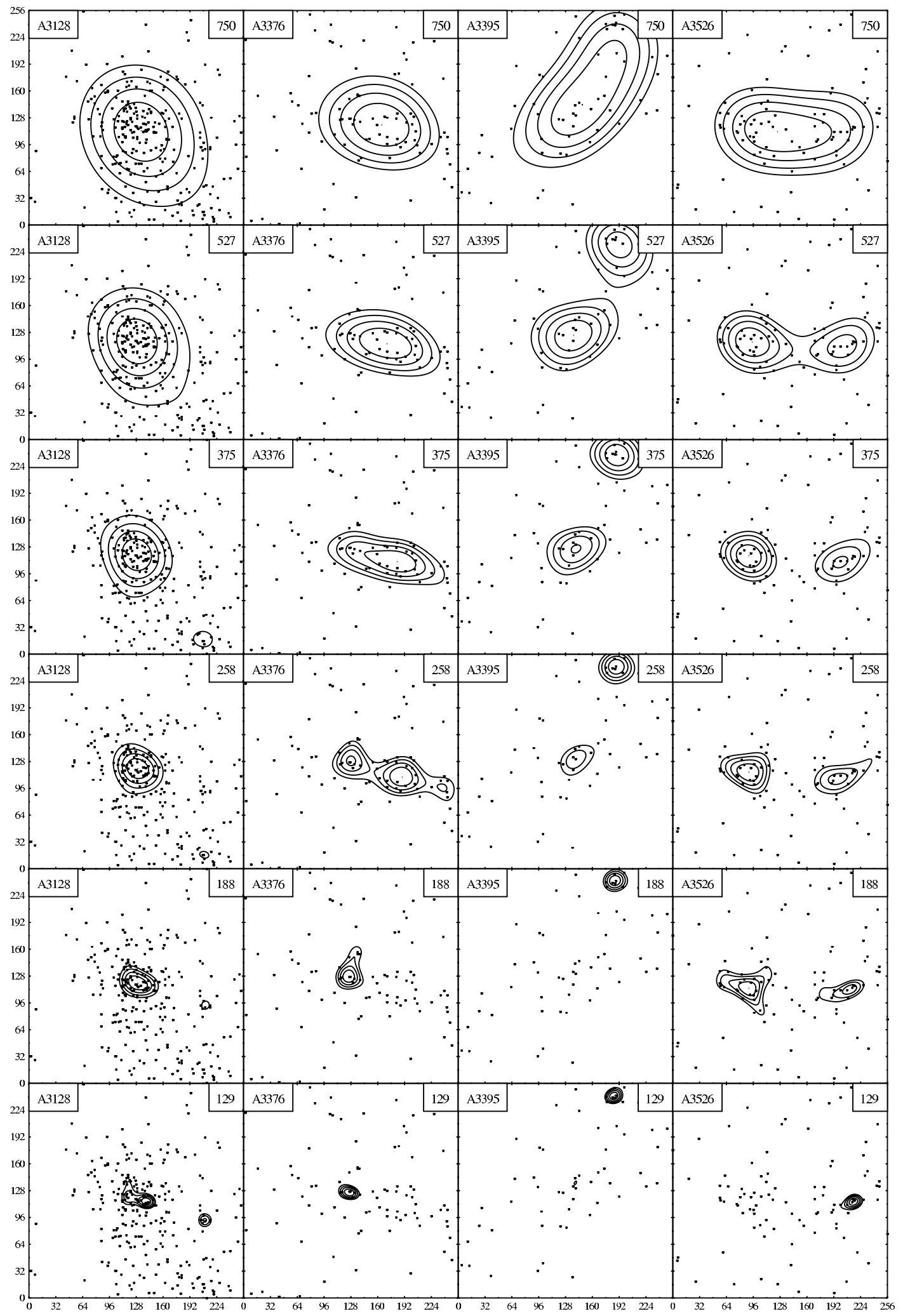

Fig. 17. Distribution of galaxies in A3128, A3376, A3395 and A3526 with wavelet images for scales $a=129,188,258,375,527,750 \mathrm{kpc}$ presented in the upper right box. 